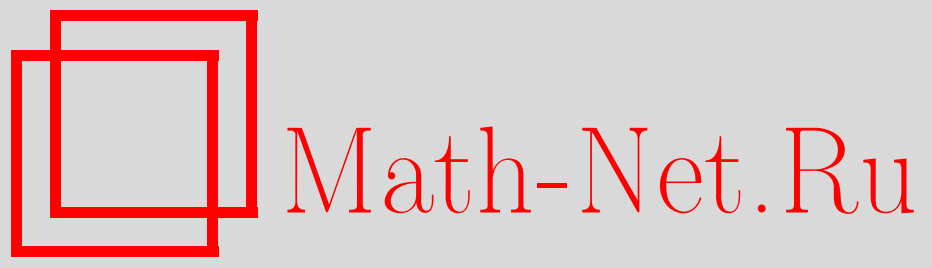

M. Grabchak, S. A. Molchanov, Limit theorems and phase transitions for two models of summation of i.i.d. random variables with a parameter, Теория вероятн. и ее примен., 2014, том 59, выпуск 2, 340-364

DOI: https://doi.org/10.4213/tvp4568

Использование Общероссийского математического портала Math-Net.Ru подразумевает, что вы прочитали и согласны с пользовательским соглашением http://www . mathnet.ru/rus/agreement

Параметры загрузки:

IP: 54.162 .85 .209

26 апреля 2023 г., 18:01:41

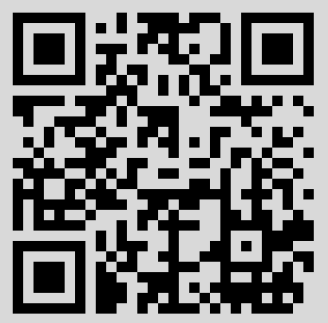




\title{
LIMIT THEOREMS AND PHASE TRANSITIONS FOR TWO MODELS OF SUMMATION OF I.I.D. RANDOM VARIABLES WITH A PARAMETER
}

\begin{abstract}
В статье рассматриваются две модели суммирования независимых одинаково распределенных случайных величин, зависящих от параметра. Первая мотивирована финансовыми приложениями, а вторая - миграцией особей. Цель исследования состоит в описании предельных законов и их бифуркаций при разных соотношениях между параметром и числом слагаемых в сумме. Мы находим, что в фазовом переходе могут появиться предельные законы, которые значительно отличаются от тех, которые присутствуют в стандартных предельных теоремах. Из наших результатов вытекает, что эти предельные законы являются лучшими моделями, по крайней мере при некоторых уровнях агрегации. Более того, мы показываем, как параметр определяет, при каких уровнях агрегации можно применять эти модели.
\end{abstract}

Ключевье слова и фразы: центральная предельная теорема, фазовый переход, модулированные устойчивые законы, контактмодели, модулированные тяжелые хвосты, натуральный масштаб.

1. Introduction. We consider two models for the summation of i.i.d. random variables with a parameter. This parameter characterizes how heavy the tails of the distribution are. While there is an infinite number of such models, we focus on those arising from particular real-world applications. The first is motivated by mathematical finance and the second by population dynamics. The first is a light tailed distribution which, in certain ways, behaves like a heavy tailed one, while the second is a heavy tailed distribution, which, in certain ways, behaves like a light tailed one. Depending on the value of the parameter, these models may act more like light tailed or more like heavy tailed distributions. We describe the asymptotic distribution of the sum under different relationships between the parameter and the number of summands.

In the case where the light tails dominate we get a Gaussian limit, and in the case where the heavy tails dominate we get an infinite variance stable distribution. Most interesting is what happens in the intermediate region, where neither regime dominates. We call this region the phase transition.

*Department of Mathematics and Statistics, University of North Carolina at Charlotte, USA; e-mail: mgrabcha@uncc.edu; smolchan@uncc.edu 
Here we get distributions that do not come up in standard limit theorems. In the first model we get a particular type of tempered stable distribution (see, e.g., [31], [4], and [19]), while in the second we get the convolution of a Gaussian and an infinite variance stable distribution. Our results were first announced in [21].

To illustrate the effects that we are interested in, we first recall the socalled random energy model (REM) introduced by Derrida in [15]. While Derrida presented the model in the context of statistical physics, we can describe it in purely mathematical terms as follows. Consider the sum

$$
S_{n}(t)=\sum_{i=1}^{n} e^{t X_{i}}
$$

where $t \in(0, \infty)$ and $\left\{X_{i}: i \geqslant 1\right\}$ are i.i.d. random variables whose moment generating function is an entire function on the complex plane. In this case $e^{t X_{1}}$ has a finite variance, and, for a fixed $t, S_{n}(t)$ will (under appropriate shifting and scaling) converge in distribution to the Gaussian. However, the situation becomes more complicated when both $n$ and $t$ approach infinity.

Intuitively, if $n$ grows «very fast» relative to $t$, then the distribution of $S_{n}(t)$ should still converge (under appropriate normalization) to the Gaussian. However, if $t$ grows «very fast» relative to $n$, then the largest term in the sum dominates and the limiting distribution is closely related to the double-exponential law from extreme value theory. Intermediate relations between $n$ and $t$ can lead to $\alpha$-stable limit laws for any $\alpha \in(0,2)$. The weak limits of $S_{n}(t)$ were characterized in [8] for the case when $X_{1}$ has a Gaussian distribution, and in [3] and [7] for the more general case when $X_{1}$ has Weibull-type tails. An extension to sums of products of exponentials, which is motivated by the nonstationary Anderson model from the theory of random media, is analyzed in [14].

Before describing our models, recall that the only possible weak limits of sums of infinitesimal triangular arrays of independent random variables are infinitely divisible distributions. The characteristic function of an infinitely divisible distribution $\mu$ can be written as $\hat{\mu}(z)=\exp \left\{C_{\mu}(z)\right\}$, where

$$
C_{\mu}(z)=-\frac{1}{2} \sigma^{2} z^{2}+i b z+\int_{\mathbf{R}}\left(e^{i z x}-1-i \frac{z x}{1+x^{2}}\right) M(\mathrm{~d} x),
$$

$\sigma^{2} \geqslant 0, b \in \mathbf{R}$, and $M$ is a Lévy measure, that is, $M$ satisfies

$$
M(\{0\})=0 \quad \text { and } \quad \int_{\mathbf{R}}\left(|x|^{2} \wedge 1\right) M(\mathrm{~d} x)<\infty
$$

The measure $\mu$ is uniquely identified by the Lévy triplet $\left(\sigma^{2}, M, b\right)$, and we write $\mu=\operatorname{ID}\left(\sigma^{2}, M, b\right)$. For details see, e.g., [24] or [32]. We also recall that 
a real-valued function $f$ on $(0, \infty)$ is called regularly varying with index $\rho$ if for any $x \in(0, \infty)$

$$
\lim _{t \rightarrow \infty} \frac{f(x t)}{f(t)}=x^{\rho} .
$$

In this case we write $f \in \mathrm{RV}_{\rho}$. For details see [6] or [30].

2. Model I: distributions with tempered heavy tails. In financial mathematics, distributions with an infinite variance are often used to model returns. While such models often provide a good fit, there is clear evidence that returns have a finite variance. Instead [22] suggests that distributions with tempered heavy tails, that is, ones that are obtained by modifying the tails of heavy tailed distributions to make them lighter, are natural models for financial returns.

One way of obtaining such distributions is to start with a heavy tailed distribution and then truncate its tails. Limit theorems for sums of i.i.d. random variables with truncated tails are characterized in [12]. We will focus on another class of models with tempered heavy tails, one where the tails are modified by the more delicate procedure of exponential tempering.

Before defining these models, we motivate the discussion by describing a simple example of a distribution with tempered heavy tails. It is based on the idea of tail truncation, but it does not quite fall into the framework considered in [12]. This simple example is used to illustrate the type of behavior that is possible.

2.1. Truncated Pareto distribution. Consider the truncated Pareto law with density

$$
p_{T}(x)= \begin{cases}c_{T} x^{-1-\alpha} & \text { if } x \in(1, T) \\ 0 & \text { otherwise }\end{cases}
$$

where $\alpha \in(0,2), T \in(1, \infty]$ is the level of truncation, and

$$
c_{T}=\frac{\alpha}{1-T^{-\alpha}}
$$

When $T=\infty$ this belongs to the domain of attraction of an $\alpha$-stable distribution. However, for $T<\infty$ the distribution is in the domain of attraction of the Gaussian. Let $S_{n}(T)=\sum_{i=1}^{n} X_{i}(T)$, where $X_{1}(T), \ldots, X_{n}(T) \stackrel{\text { iid }}{\sim} p_{T}$. We are interested in what happens when both $n$ and $T$ approach infinity. Intuitively, if $n$ approaches infinity much faster than $T$ then the limit should still be Gaussian, but when $T$ approaches infinity much faster than $n$ the limit should be $\alpha$-stable. We now characterize the weak limits of $S_{n}(T)$.

Theorem 1. Let $T_{n} \rightarrow \infty$ such that $T_{n}<\infty$ for each $n$, and assume that $n^{-1 / \alpha} T_{n} \rightarrow c \in[0, \infty]$. 
1. If $c \in(0, \infty]$, then

$$
n^{-1 / \alpha} S_{n}\left(T_{n}\right)-\kappa_{n} \stackrel{d}{\rightarrow} R_{\alpha, c},
$$

where

$$
\kappa_{n}= \begin{cases}n^{1-1 / \alpha} \frac{\alpha}{\alpha-1} & \text { if } \alpha \in(1,2), \\ \ln n & \text { if } \alpha=1, \\ 0 & \text { if } \alpha \in(0,1),\end{cases}
$$

$R_{\alpha, c} \sim \operatorname{ID}\left(0, H_{\alpha, c}, \gamma^{\prime}\right)$ for some $\gamma^{\prime} \in \mathbf{R}$, and

$$
H_{\alpha, c}(\mathrm{~d} x)=\alpha x^{-\alpha-1} 1_{\{0<x<c\}} \mathrm{d} x .
$$

2. If $c=0$, then

$$
b_{n} S_{n}\left(T_{n}\right)-\kappa_{n}^{\prime} \stackrel{d}{\rightarrow} N\left(0, \sigma^{2}\right),
$$

where $\sigma^{2}=\alpha /(2-\alpha), b_{n}=n^{-1 / 2} T_{n}^{-(2-\alpha) / 2}$, and

$$
\kappa_{n}^{\prime}= \begin{cases}n b_{n} \frac{T_{n}^{1-\alpha}-1}{1-T_{n}^{-\alpha}} \frac{\alpha}{1-\alpha} & \text { if } \alpha \neq 1, \\ n b_{n} \frac{\ln T_{n}}{1-T_{n}^{-1}} & \text { if } \alpha=1 .\end{cases}
$$

The next result gives a law of large numbers for this situation.

Theorem 2. Let $T_{n} \rightarrow \infty$ such that $T_{n}<\infty$ for each $n$, and assume that $n^{-1 / \alpha} T_{n} \rightarrow c \in[0, \infty]$.

1. If $\alpha \in(1,2)$, then

$$
\frac{S_{n}\left(T_{n}\right)}{n} \stackrel{p}{\rightarrow} \frac{\alpha}{\alpha-1} .
$$

2. If $\alpha=1$ and $c \in(0, \infty]$, then

$$
\frac{S_{n}\left(T_{n}\right)}{n \ln n} \stackrel{p}{\rightarrow} 1 .
$$

3. If $\alpha \in(0,1)$ and $c \in(0, \infty]$, then for any $\left\{a_{n}\right\}$ with $n^{1 / \alpha} a_{n} \rightarrow 0$

$$
a_{n} S_{n}\left(T_{n}\right) \stackrel{p}{\rightarrow} 0
$$

4. If $\alpha \in(0,1]$ and $c=0$, then

$$
\begin{array}{ll}
\frac{S_{n}\left(T_{n}\right)}{n T_{n}^{1-\alpha}} \stackrel{p}{\rightarrow} \frac{\alpha}{1-\alpha} & \text { if } \alpha \in(0,1), \\
\frac{S_{n}\left(T_{n}\right)}{n \ln T_{n}} \stackrel{p}{\rightarrow} 1 & \text { if } \alpha=1 .
\end{array}
$$

Note that when $\alpha \in(1,2)$ and $X \sim p_{\infty}$, then $\mathbf{E}[X]=\alpha /(\alpha-1)$. Thus, in this case, the truncation has no effect on the limit. 


\subsection{Exponentially tempered infinitely divisible distributions.} In this subsection we consider a class of infinitely divisible distributions with tempered heavy tails. It is well known that the tails of infinitely divisible distributions are intimately related with the tails of their Lévy measures. In fact, an infinitely divisible distribution has an infinite variance if and only if $\int_{\mathbf{R}} x^{2} M(\mathrm{~d} x)=\infty$. This suggests that if we wish to make the tails lighter we can modify the Lévy measure as follows.

Let $\mu=\mathrm{ID}\left(\sigma^{2}, M, b\right)$ be an infinitely divisible distribution in the domain of attraction of some infinite variance $\alpha$-stable law. For $p, \ell \in(0, \infty)$ define

$$
M_{p}^{(\ell)}(\mathrm{d} x)=e^{-|x|^{p} / \ell} M(\mathrm{~d} x) \text { and } \mu_{p}^{(\ell)}=\operatorname{ID}\left(\sigma^{2}, M_{p}^{(\ell)}, b\right) .
$$

We call this exponential tempering of the infinitely divisible distribution. Clearly, $\mu_{p}^{(\ell)}$ is now in the domain of attraction of the Gaussian. However, if $\ell$ is very large, then $M_{p}^{(\ell)}$ will be similar to $M$ in some (large) central region. This will imply that $\mu_{p}^{(\ell)}$ will be similar to $\mu$ in some central region as well, but very large jumps will be «tempered».

When $p=1, \sigma^{2}=0$, and $M((-\infty, 0])=0$, the relationship between $\mu$ and $\mu_{1}^{(\ell)}$ can be given explicitly. If $\mu$ has Lebesgue density $f$, then $\mu_{1}^{(\ell)}$ has Lebesgue density

$$
K_{\ell} e^{-x / \ell} f\left(x-\gamma_{\ell}\right)
$$

for some constants $K_{\ell}, \gamma_{\ell}$. In this case $\mu_{1}^{(\ell)}$ is sometimes called the Esscher transform of $\mu$. For details and applications of Esscher transforms to finance see $[13]$.

Since, for the applications of interest, $\ell$ is very large, we consider the asymptotic behavior of sums of $n$ i.i.d. random variables with distribution $\mu_{p}^{(\ell)}$ as both $n$ and $\ell$ approach infinity. We assume that $p>0$ is fixed. As we will see, the parameter $\ell$ plays a role analogous the one played by $T$ in the case of the truncated Pareto distribution.

Without loss of generality, we restrict our attention to the case $\mu=$ ID $(0, M, 0)$, where $M((-\infty, 0])=0$. The more general situation follows by looking at each part separately. Assume that $\mu$ is in the domain of attraction of some $\alpha$-stable distribution with $\alpha \in(0,2)$. Equivalently, this means that there is a function $L \in \mathrm{RV}_{0}$ such that

$$
M(\{x: x>t\})=t^{-\alpha} L(t) .
$$

Let

$$
V(t)=\frac{t^{\alpha}}{L(t)} \quad \text { and } \quad a_{t}=\frac{1}{V^{\leftarrow}(t)},
$$

where

$$
V^{\leftarrow}(t)=\inf \{s: V(s)>t\}
$$


By Theorem 1.5.12 in [6] $a \in \mathrm{RV}_{-1 / \alpha}$. If $X_{1}, X_{2}, \ldots \stackrel{\text { iid }}{\sim} \mu$, then there is a sequence $\left\{\eta_{n}\right\}$ in $\mathbf{R}$ with

$$
a_{n} \sum_{i=1}^{n}\left(X_{i}-\eta_{n}\right) \stackrel{d}{\rightarrow} R_{\alpha, \infty}
$$

where $R_{\alpha, \infty} \sim \operatorname{ID}\left(0, M_{\infty}, 0\right)$ is an $\alpha$-stable random variable with

$$
M_{\infty}(\mathrm{d} x)=\alpha x^{-1-\alpha} 1_{\{x>0\}} \mathrm{d} x .
$$

Fix $p>0$ and let $\left\{\ell_{n}\right\}$ be a sequence of positive real numbers such that $\ell_{n} \rightarrow \infty$. Define $M_{p}^{\left(\ell_{n}\right)}$ and $\mu_{p}^{\left(\ell_{n}\right)}$ as in (3) and set

$$
S_{n}\left(\ell_{n}\right)=\sum_{i=1}^{n} X_{i}\left(\ell_{n}\right)
$$

where $X_{1}\left(\ell_{n}\right), \ldots, X_{n}\left(\ell_{n}\right) \stackrel{\text { iid }}{\sim} \mu_{p}^{\left(\ell_{n}\right)}$. We now give our main results for this model.

Theorem 3. 1. If $a_{n}^{p} \ell_{n} \rightarrow c \in(0, \infty]$, then $a_{n} S_{n}\left(\ell_{n}\right)-\kappa_{n} \stackrel{d}{\rightarrow} R_{\alpha, c}$, where

$$
\kappa_{n}=n a_{n} \int_{0}^{\infty} \frac{x^{3}}{\left(1+x^{2}\right)\left(1+x^{2} a_{n}^{2}\right)} e^{-x^{p} / \ell_{n}} M(\mathrm{~d} x)
$$

and $R_{\alpha, c} \sim \operatorname{ID}\left(0, H_{\alpha, c}, 0\right)$ with

$$
H_{\alpha, c}(\mathrm{~d} x)=e^{-x^{p} / c} M_{\infty}(\mathrm{d} x)=\alpha e^{-x^{p} / c} x^{-1-\alpha} 1_{\{x>0\}} \mathrm{d} x .
$$

2. If $a_{n}^{p} \ell_{n} \rightarrow 0$, then $b_{n} S_{n}\left(\ell_{n}\right)-\kappa_{n}^{\prime} \stackrel{d}{\rightarrow} N\left(0, \sigma^{2}\right)$, where

$$
\begin{aligned}
\sigma^{2} & =\frac{\alpha}{p} \Gamma\left(\frac{2-\alpha}{p}\right) \\
b_{n} & =n^{-1 / 2} \ell_{n}^{-1 / p} \sqrt{V\left(\ell_{n}^{1 / p}\right)}=n^{-1 / 2} \ell_{n}^{-(2-\alpha) /(2 p)}\left[L\left(\ell_{n}^{1 / p}\right)\right]^{-1 / 2}, \\
\kappa_{n}^{\prime} & =n b_{n} \int_{0}^{\infty} \frac{x^{3}}{\left(1+x^{2}\right)\left(1+x^{2} b_{n}^{2}\right)} e^{-x^{p} / \ell_{n}} M(\mathrm{~d} x) .
\end{aligned}
$$

R e $\mathrm{m}$ a r k 1 . The distribution ID $\left(0, H_{\alpha, c}, 0\right)$ with $H_{\alpha, c}$ given by (10) and $c \in(0, \infty)$ is a particular type of tempered stable distribution, which is sometimes called an elementary tempered stable distribution. For more information on tempered stable distributions see [31], [4], [19], and [20]. In [18] it is shown that elementary tempered stable distributions are the building blocks from which all other tempered stable distributions can be constructed. Such distributions have appeared in other limit theorems, see [11] and [26].

$\mathrm{R}$ e $\mathrm{m}$ a $\mathrm{rk} 2$. In practice, for instance when modeling financial returns, the parameter $\ell_{n}$ is not actually approaching infinity. Instead it is 
some fixed but (very) large constant $\ell$. We can write $a_{n}^{p} \ell=\left[n^{-1} \ell^{\alpha / p}\right]^{p / \alpha} L^{\prime}(n)$ for some $L^{\prime} \in \mathrm{RV}_{0}$. If $S_{n}(\ell)$ is the sum of $n$ i.i.d. random variables with distribution $\mu_{p}^{(\ell)}$, then Theorem 3 can be interpreted as follows. When $n$ is large but much smaller than $\ell^{\alpha / p}$ the distribution of $S_{n}(\ell)$ is close to a stable distribution. When it is on the order of $\ell^{\alpha / p}$, the distribution is close to a tempered stable distribution. However, once $n$ is much larger than $\ell^{\alpha / p}$ the distribution of $S_{n}(\ell)$ is well approximated by the Gaussian. A constant which determines when such regimes occur was called a «natural scale» in [22]. Thus, in this case, the natural scale is given by $\ell^{\alpha / p}$. Similarly, the natural scale for the truncated Pareto distribution is $T^{\alpha}$. An approach to approximating the error in such approximations is given in [22].

We now give necessary and sufficient conditions for the law of large numbers to hold in this situation.

Theorem 4. 1. If $\alpha \in(1,2)$, then

$$
\frac{S_{n}\left(\ell_{n}\right)}{n} \stackrel{p}{\rightarrow} \int_{0}^{\infty} \frac{x^{3}}{1+x^{2}} M(\mathrm{~d} x) .
$$

2. If $\alpha=1$ and $a_{n}^{p} \ell_{n} \rightarrow c \in(0, \infty]$, then $\kappa_{n} \rightarrow \infty$ and

$$
\frac{a_{n} S_{n}\left(\ell_{n}\right)}{\kappa_{n}} \stackrel{p}{\rightarrow} 1 .
$$

3. If $\alpha \in(0,1)$ and $a_{n}^{p} \ell_{n} \rightarrow c \in(0, \infty]$, then for any $\left\{a_{n}^{\prime}\right\}$ with $\frac{a_{n}^{\prime}}{/} a_{n} \rightarrow 0$

$$
a_{n}^{\prime} S_{n}\left(\ell_{n}\right) \stackrel{p}{\rightarrow} 0 .
$$

4. If $\alpha \in(0,1]$ and $a_{n}^{p} \ell_{n} \rightarrow 0$, then $\kappa_{n}^{\prime} \rightarrow \infty$ and

$$
\frac{b_{n} S_{n}\left(\ell_{n}\right)}{\kappa_{n}^{\prime}} \stackrel{p}{\rightarrow} 1 \text {. }
$$

Note that when $\alpha \in(1,2)$ and $X \sim \operatorname{ID}(0, M, 0)$, then $\mathbf{E}[X]=$ $\int_{0}^{\infty} x^{3}\left(1+x^{2}\right)^{-1} M(\mathrm{~d} x)$. Thus, in this case, the exponential tempering has no effect on the limit.

2.3. Exponentially tempered distributions. There are wellknown results relating convergence of sequences of sums of infinitely divisible distributions to sequences of sums of infinitesimal triangular arrays. In this section we use such results to extend our results from subsection 2.2.

Let $\mu$ be a probability measure in the domain of attraction of some infinite variance $\alpha$-stable law. Without loss of generality, we assume that $\mu((-\infty, 0])=0$. For $p, \ell \in(0, \infty)$ define the tempered probability measure

$$
\mu_{p}^{(\ell)}(\mathrm{d} x)=c_{\ell} e^{-x^{p} / \ell} \mu(\mathrm{d} x),
$$


where

$$
c_{\ell}=\left[\int_{0}^{\infty} e^{-x^{p} / \ell} \mu(\mathrm{d} x)\right]^{-1} .
$$

Note that, although we use the same notation as in the previous subsection, $\mu_{p}^{(\ell)}$ means something quite different here than what it meant there.

We will consider the asymptotic behavior of sums of $n$ i.i.d. random variables with distribution $\mu_{p}^{(\ell)}$ as both $n$ and $\ell$ approach infinity. Since $\mu$ is in the domain of attraction of an $\alpha$-stable distribution with $\alpha \in(0,2)$, there exists a function $L \in \mathrm{RV}_{0}$ such that

$$
\mu(\{x: x>t\})=t^{-\alpha} L(t) .
$$

As in the previous subsection let

$$
V(t)=\frac{t^{\alpha}}{L(t)} \quad \text { and } \quad a_{t}=\frac{1}{V^{\leftarrow}(t)},
$$

where $V^{\leftarrow}(t)=\inf \{s: V(s)>t\}$. If $X_{1}, X_{2}, \ldots \stackrel{\text { iid }}{\sim} \mu$, then there is a sequence $\left\{\eta_{n}\right\}$ in $\mathbf{R}$ with

$$
a_{n} \sum_{i=1}^{n}\left(X_{i}-\eta_{n}\right) \stackrel{d}{\rightarrow} R_{\alpha, \infty}
$$

where $R_{\alpha, \infty} \sim \operatorname{ID}\left(0, M_{\infty}, 0\right)$ and $M_{\infty}$ is as in (8). Fix $p>0$ and let $\left\{\ell_{n}\right\}$ be a sequence of positive real numbers such that $\ell_{n} \rightarrow \infty$. Define $\mu_{p}^{\left(\ell_{n}\right)}$ as in (11) and set

$$
S_{n}\left(\ell_{n}\right)=\sum_{i=1}^{n} X_{i}\left(\ell_{n}\right)
$$

where $X_{1}\left(\ell_{n}\right), \ldots, X_{n}\left(\ell_{n}\right) \stackrel{\text { iid }}{\sim} \mu_{p}^{\left(\ell_{n}\right)}$. We now give our main results for this model.

Theorem 5. 1. If $a_{n}^{p} \ell_{n} \rightarrow c \in(0, \infty]$, then

$$
a_{n} S_{n}\left(\ell_{n}\right)-\kappa_{n} \stackrel{d}{\rightarrow} R_{\alpha, c}+\eta_{\alpha, c}
$$

where

$$
\begin{aligned}
\kappa_{n} & =c_{\ell_{n}} n a_{n} \int_{0}^{1 / a_{n}} x e^{-x^{p} / \ell_{n}} \mu(\mathrm{d} x), \\
\eta_{\alpha, c} & =\int_{0}^{\infty} x\left(\frac{1}{1+|x|^{2}}-1_{\{x<1\}}\right) H_{\alpha, c}(\mathrm{~d} x),
\end{aligned}
$$

$R_{\alpha, c} \sim \mathrm{ID}\left(0, H_{\alpha, c}, 0\right)$, and

$$
H_{\alpha, c}(\mathrm{~d} x)=e^{-x^{p} / c} M_{\infty}(\mathrm{d} x)=\alpha e^{-x^{p} / c} x^{-1-\alpha} 1_{\{x>0\}} \mathrm{d} x .
$$


2. If $a_{n}^{p} \ell_{n} \rightarrow 0$, then $b_{n} S_{n}\left(\ell_{n}\right)-\kappa_{n}^{\prime} \stackrel{d}{\rightarrow} N\left(0, \sigma^{2}\right)$, where

$$
\begin{aligned}
\sigma^{2} & =\frac{\alpha}{p} \Gamma\left(\frac{2-\alpha}{p}\right) \\
b_{n} & =n^{-1 / 2} \ell_{n}^{-1 / p} \sqrt{V\left(\ell_{n}^{1 / p}\right)}=n^{-1 / 2} \ell_{n}^{-(2-\alpha) /(2 p)}\left[L\left(\ell_{n}^{1 / p}\right)\right]^{-1 / 2}, \\
\kappa_{n}^{\prime} & =c_{\ell_{n}} n b_{n} \int_{0}^{1 / b_{n}} x e^{-x^{p} / \ell_{n}} \mu(\mathrm{d} x) .
\end{aligned}
$$

We now give necessary and sufficient conditions the law of large numbers to hold.

Theorem 6. 1. If $\alpha \in(1,2)$, then

$$
\frac{S_{n}\left(\ell_{n}\right)}{n} \stackrel{p}{\rightarrow} \int_{0}^{\infty} x \mu(\mathrm{d} x) .
$$

2. If $\alpha=1$ and $a_{n}^{p} \ell_{n} \rightarrow c \in(0, \infty]$, then $\kappa_{n} \rightarrow \infty$ and

$$
\frac{a_{n} S_{n}\left(\ell_{n}\right)}{\kappa_{n}} \stackrel{p}{\rightarrow} 1 \text {. }
$$

3. If $\alpha \in(0,1)$ and $a_{n}^{p} \ell_{n} \rightarrow c \in(0, \infty]$, then for any $\left\{a_{n}^{\prime}\right\}$ with $\frac{a_{n}^{\prime}}{/} a_{n} \rightarrow 0$

$$
a_{n}^{\prime} S_{n}\left(\ell_{n}\right) \stackrel{p}{\rightarrow} 0
$$

4. If $\alpha \in(0,1]$ and $a_{n}^{p} \ell_{n} \rightarrow 0$, then $\kappa_{n}^{\prime} \rightarrow \infty$ and

$$
\frac{b_{n} S_{n}\left(\ell_{n}\right)}{\kappa_{n}^{\prime}} \stackrel{p}{\rightarrow} 1 .
$$

3. Model II: light tails with a heavy tailed impurity. A popular model of population dynamics is the so-called contact model on $\mathbf{R}^{d}$. This model is a branching process where an offspring migrates from the parent's location $x$ to a new position $x+z$ following some distribution $F$. For details see [17], [27], and the references therein.

When $d \geqslant 3,[27]$ showed that if $F$ has light tails - that is, if it is in the domain of attraction of the Gaussian - then the number of particles at every point of $\mathbf{R}^{d}$ approaches a limiting distribution. However, in [17] it was shown that when $d \in\{1,2\}$ a limiting distribution can only exist when $F$ is in the domain of attraction of an infinite variance stable distribution. Thus, for the distribution of species to reach equilibrium in one or two dimensions (the most relevant for ecological applications), the distribution of their migration needs to be heavy tailed. In practice, however, most species do not migrate very far. The biological data indicates that the heavy tails are related to a small number of «very active» species in the population. This suggests that 
$F$ is actually a mixture of a distribution with light tails and one with heavy tails. That is,

$$
F(x)=F_{p}(x)=(1-p) F_{1}(x)+p F_{2}(x),
$$

where $p$ is the small number corresponding to the proportion of «very active» species, and $F_{1}$ and $F_{2}$ are, respectively, distributions in the domain of attraction of the Gaussian and of an infinite variance stable law. Such distributions were used in [10] to model the dispersal of seeds and in [9] to model the travel patterns of humans. Since we are interested in the case where $p$ is very small, we will consider the asymptotic behavior of sums of $n$ i.i.d. random variables with distribution $F_{p}$ as $n$ approaches infinity and $p$ approaches zero. Before proceeding, we note that the problem of approximating sums of random variables with distributions of the form $F_{p}$ goes back to [28] and [25]. More recent results can be found in [33]-[36].

We now formulate our problem in a slightly more general context. Fix $0<\alpha_{2}<\alpha_{1} \leqslant 2$. Let $F_{1}$ and $F_{2}$ be distributions, not concentrated at a point, such that $F_{1}$ is in the domain of attraction of some $\alpha_{1}$-stable random variable $R_{\alpha_{1}}$ and $F_{2}$ is in the domain of attraction of some $\alpha_{2}$-stable random variable $R_{\alpha_{2}}$. For simplicity, if $\alpha_{j}=1(j=1,2)$ we make the additional assumption that $F_{j}$ is symmetric around zero.

Let $\left\{X_{i}\right\}$ and $\left\{Y_{i}\right\}$ be sequences of i.i.d. random variables with distributions $F_{1}$ and $F_{2}$, respectively. The above assumptions imply that there is a function $c_{\alpha_{1}} \in \mathrm{RV}_{-1 / \alpha_{1}}$ such that

$$
c_{\alpha_{1}}(n) \sum_{i=1}^{n}\left(X_{i}-b_{\alpha_{1}}\right) \stackrel{d}{\rightarrow} R_{\alpha_{1}},
$$

where

$$
b_{\alpha_{1}}= \begin{cases}0, & \alpha_{1} \in(0,1] \\ \mathbf{E}\left[X_{1}\right] & \alpha_{1} \in(1,2] .\end{cases}
$$

The corresponding result holds for the sequence $\left\{Y_{i}\right\}$ if we replace $\alpha_{1}$ by $\alpha_{2}$.

Let $\left\{p_{n}\right\}$ be a sequence in $(0,1)$ with $p_{n} \rightarrow 0$. Assume that $\left\{X_{i}\right\}$ and $\left\{Y_{i}\right\}$ are independent of each other and let $B_{1}^{(n)}, \ldots, B_{n}^{(n)}$ be i.i.d. Bernoulli random variables independent of both $\left\{X_{i}\right\}$ and $\left\{Y_{i}\right\}$ such that

$$
\mathbf{P}\left(B_{i}^{(n)}=1\right)=p_{n}, \quad \mathbf{P}\left(B_{i}^{(n)}=0\right)=1-p_{n}
$$

Consider the triangular array

$$
Z_{i}^{(n)}=\left(1-B_{i}^{(n)}\right) X_{i}+B_{i}^{(n)} Y_{i}, \quad i=1, \ldots, n .
$$

By relabeling the indices it is clear that

$$
\sum_{i=1}^{n} Z_{i}^{(n)} \stackrel{d}{=} \sum_{i=N_{n}+1}^{n} X_{i}+\sum_{i=1}^{N_{n}} Y_{i}
$$


where $N_{n} \sim B\left(n, p_{n}\right)$ is independent of the sequences $\left\{X_{i}\right\}$ and $\left\{Y_{i}\right\}$. Clearly, the distribution of $Z_{i}^{(n)}$ is $F_{p_{n}}$. We now state our main results for this model.

Theorem 7. 1. If $p_{n} n \rightarrow \eta \in[0, \infty)$, then

$$
c_{\alpha_{1}}(n) \sum_{i=1}^{n}\left(Z_{i}^{(n)}-b_{\alpha_{1}}\right) \stackrel{d}{\rightarrow} R_{\alpha_{1}} .
$$

2. If $p_{n} n \rightarrow \infty$ and $c_{\alpha_{1}}(n) / c_{\alpha_{2}}\left(p_{n} n\right) \rightarrow 0$, then

$$
c_{\alpha_{1}}(n) \sum_{i=1}^{n}\left(Z_{i}^{(n)}-b_{\alpha_{1}}-p_{n}\left(b_{\alpha_{2}}-b_{\alpha_{1}}\right)\right) \stackrel{d}{\rightarrow} R_{\alpha_{1}} .
$$

3. If $p_{n} n \rightarrow \infty$ and $c_{\alpha_{1}}(n) / c_{\alpha_{2}}\left(p_{n} n\right) \rightarrow \kappa \in(0, \infty]$, then

$$
c_{\alpha_{2}}\left(p_{n} n\right) \sum_{i=1}^{n}\left(Z_{i}^{(n)}-b_{\alpha_{1}}-p_{n}\left(b_{\alpha_{2}}-b_{\alpha_{1}}\right)\right) \stackrel{d}{\rightarrow} \kappa^{-1} R_{\alpha_{1}}+R_{\alpha_{2}},
$$

where $R_{\alpha_{1}}$ and $R_{\alpha_{2}}$ are independent.

$\mathrm{R}$ e m a r k 3 . When both $F_{1}$ and $F_{2}$ belong to the domain of normal attraction of the corresponding stable distribution, that is, when $c_{\alpha_{1}}(t)=$ $t^{-1 / \alpha_{1}}$ and $c_{\alpha_{2}}(t)=t^{-1 / \alpha_{2}}$, we have

$$
\frac{c_{\alpha_{1}}(n)}{c_{\alpha_{2}}\left(p_{n} n\right)}=n^{1 / \alpha_{2}-1 / \alpha_{1}} p_{n}^{1 / \alpha_{2}}=\left(n^{1-\alpha_{2} / \alpha_{1}} p_{n}\right)^{1 / \alpha_{2}} .
$$

In this case, the condition $c_{\alpha_{1}}(n) / c_{\alpha_{2}}\left(p_{n} n\right) \rightarrow \kappa \in[0, \infty]$ is equivalent to the condition $n^{1-\alpha_{2} / \alpha_{1}} p_{n} \rightarrow \kappa^{\alpha_{2}}$.

We now give a law of large numbers.

Theorem 8. Assume that $\alpha_{1} \in(1,2]$.

1. If $n p_{n} \rightarrow \eta \in[0, \infty)$, then

$$
\frac{1}{n} \sum_{i=1}^{n} Z_{i}^{(n)} \stackrel{p}{\rightarrow} \mathbf{E}\left[X_{1}\right]
$$

2. If $n p_{n} \rightarrow \infty$ and $n c_{\alpha_{2}}\left(n p_{n}\right) \rightarrow \kappa \in(0, \infty]$, then

$$
\frac{1}{n} \sum_{i=1}^{n} Z_{i}^{(n)} \stackrel{d}{\rightarrow} \mathbf{E}\left[X_{1}\right]+\kappa^{-1} R_{\alpha_{2}} .
$$

3. If $n p_{n} \rightarrow \infty$ and $n c_{\alpha_{2}}\left(n p_{n}\right) \rightarrow 0$, then $c_{\alpha_{1}}(n) / c_{\alpha_{2}}\left(p_{n} n\right) \rightarrow \infty$ and for any $\left\{a_{n}\right\}$ with

$$
\lim _{n \rightarrow \infty} \frac{a_{n}}{c_{\alpha_{2}}\left(n p_{n}\right)}=0
$$

we have

$$
a_{n} \sum_{i=1}^{n} Z_{i}^{(n)} \stackrel{p}{\rightarrow} 0
$$


R e m a r k 4. Note that $\alpha_{1} \in(1,2]$ implies that $\mathbf{E}\left|X_{1}\right|<\infty$, and when $\alpha_{2} \in(1,2)$ we always have either $n p_{n} \rightarrow \eta \in[0, \infty)$ or $n p_{n} \rightarrow \infty$ and $n c_{\alpha_{2}}\left(n p_{n}\right) \rightarrow \infty$.

$\mathrm{R} \mathrm{e} \mathrm{m} \mathrm{a} \mathrm{r} \mathrm{k} 5$. When both $F_{1}$ and $F_{2}$ belong to the domain of normal attraction of the corresponding stable distribution, we have

$$
n c_{\alpha_{2}}\left(n p_{n}\right)=n^{1-1 / \alpha_{2}} p_{n}^{-1 / \alpha_{2}}=\left(n^{\alpha_{2}-1} p_{n}^{-1}\right)^{1 / \alpha_{2}} .
$$

In this case the condition $n c_{\alpha_{2}}\left(p_{n} n\right) \rightarrow \kappa \in[0, \infty]$ is equivalent to the condition $n^{\alpha_{2}-1} p_{n}^{-1} \rightarrow \kappa^{\alpha_{2}}$.

$\mathrm{R} \mathrm{e} \mathrm{m}$ a r k 6. By the Cramér-Wold device, it is straightforward to extend Theorems 7 and 8 to the multivariate case.

\section{Proofs for subsection 2.1.}

Pr o of of $\mathrm{Th}$ e o r e m 1 . The first part follows by a straightforward application of standard results for convergence in distribution of the sums of infinitesimal triangular arrays to infinitely divisible distribution. See, e.g., [24, Theorem 1.7.3] or [29, Theorem 3.2.2]. The second part follows by checking that Lyapounov's condition holds, see, e.g., [5, Section 27]. Theorem 1 is proved.

Proof of $\mathrm{T}$ h e ore $\mathrm{m} 2$. Fix $\alpha \in(1,2)$. If $c \in(0, \infty]$, then by Theorem 1 and Slutsky's theorem

$$
\begin{aligned}
\frac{S_{n}\left(T_{n}\right)}{n} & =\frac{n^{-1 / \alpha} S_{n}\left(T_{n}\right)-\kappa_{n}}{n^{1-1 / \alpha}}+\frac{\kappa_{n}}{n^{1-1 / \alpha}} \\
& =\frac{n^{-1 / \alpha} S_{n}\left(T_{n}\right)-\kappa_{n}}{n^{1-1 / \alpha}}+\frac{\alpha}{\alpha-1} \stackrel{p}{\rightarrow} \frac{\alpha}{\alpha-1} .
\end{aligned}
$$

If $c=0$ the proof is similar, we just need to show that $n b_{n} \rightarrow \infty$. To see this note that $(2-\alpha) / \alpha<1$ thus $T_{n}^{(2-\alpha) / \alpha}<T_{n}$ and hence

$$
n b_{n}=\left(n^{-1 / \alpha} T_{n}^{(2-\alpha) / \alpha}\right)^{-\alpha / 2} \geqslant\left(n^{-1 / \alpha} T_{n}\right)^{-\alpha / 2} \rightarrow \infty .
$$

Similarly, when $\alpha=1$ and $c \in(0, \infty]$ Theorem 1 and Slutsky's Theorem imply that

$$
\frac{S_{n}\left(T_{n}\right)}{n \ln n}=\frac{n^{-1} S_{n}\left(T_{n}\right)-\ln n}{\ln n}+1 \stackrel{p}{\rightarrow} 1 .
$$

When $\alpha \in(0,1)$ and $c \in(0, \infty]$ Theorem 1 and Slutsky's theorem imply that

$$
a_{n} S_{n}\left(T_{n}\right)=a_{n} n^{1 / \alpha} n^{-1 / \alpha} S_{n}\left(T_{n}\right) \stackrel{p}{\rightarrow} 0 .
$$

When $\alpha \in(0,1]$ and $c=0$ the result follows by arguments similar to the previous cases. Theorem 2 is proved. 
5. Proofs for subsection 2.2. First, we recall the definition of vague convergence of Lévy measures.

$\mathrm{D}$ e f i n i t i o n 1 . Let $M, M_{1}, M_{2}, \ldots$ be a sequence of Borel measures on $\mathbf{R}$ satisfying (1). We write $M_{n} \stackrel{v}{\rightarrow} M$ if for every bounded continuous function $f$ vanishing on a neighborhood of 0

$$
\lim _{n \rightarrow \infty} \int_{\mathbf{R}} f(x) M_{n}(\mathrm{~d} x)=\int_{\mathbf{R}} f(x) M(\mathrm{~d} x) .
$$

Several statements that are equivalent to this definition are given by the Portmanteau theorem, given in, e.g., [2]. The following result is fundamental to our proof, it is a version of [29, Theorem 3.1.16] or [32, Theorem 8.7].

Proposition 1. Let $\mu_{n}=\mathrm{ID}\left(\sigma_{n}^{2}, M_{n}, b_{n}\right)$ for $n=1,2, \ldots$. If there is a probability measure $\mu$ such that $\mu_{n} \stackrel{w}{\rightarrow} \mu$, then $\mu=\operatorname{ID}\left(\sigma^{2}, M, b\right)$. Moreover, $\mu_{n} \stackrel{w}{\rightarrow} \mu$ if and only if $b_{n} \rightarrow b, M_{n} \stackrel{v}{\rightarrow} M$, and

$$
\sigma^{2}=\lim _{\varepsilon \downarrow 0} \lim _{n \rightarrow \infty}\left(\sigma_{n}^{2}+\int_{|x| \leqslant \varepsilon} x^{2} M_{n}(\mathrm{~d} x)\right) .
$$

Let $M(\mathrm{~d} x)$ and $L(t)$ be as in (4), let $V(t)$ and $a_{t}$ be given by (5), let $\mu=$ ID $(0, M, 0)$, and let $L_{1}(t)=[L(t)]^{-1 / \alpha}$. Then $L_{1} \in \mathrm{RV}_{0}, V(t)=t^{\alpha}\left[L_{1}(t)\right]^{\alpha}$, and by Proposition 1.5.15 in [6] $1 / a_{t}=t^{1 / \alpha} L_{1}^{\#}\left(t^{1 / \alpha}\right)$, where $L_{1}^{\#}$ is a de Bruijn conjugate of $L_{1}$. This means that

$$
\lim _{n \rightarrow \infty} L_{1}^{\#}\left(n^{1 / \alpha}\right) L_{1}\left(\frac{1}{a_{n}}\right)=\lim _{n \rightarrow \infty} L_{1}^{\#}\left(n^{1 / \alpha}\right) L_{1}\left(n^{1 / \alpha} L_{1}^{\#}\left(n^{1 / \alpha}\right)\right)=1 .
$$

Theorem 1.5.12 in [6] implies that

$$
V\left(\frac{1}{a_{t}}\right) \sim t \quad \text { as } t \rightarrow \infty .
$$

The Lévy measure of $a_{n} \sum_{i=1}^{n} X_{i}$, where $X_{1}, X_{2}, \ldots, X_{n} \stackrel{\text { iid }}{\sim} \mu$, is given by

$$
M_{n}(A)=n \int_{0}^{\infty} 1_{A}\left(a_{n} x\right) M(\mathrm{~d} x), \quad A \in \mathfrak{B}(\mathbf{R}) .
$$

Proposition 1 and (7) imply that

$$
M_{n} \stackrel{v}{\rightarrow} M_{\infty} \quad \text { and } \quad \lim _{\varepsilon \downarrow 0} \lim _{n \rightarrow \infty} \int_{0}^{\varepsilon} x^{2} M_{n}(\mathrm{~d} x)=0 .
$$

Lemma 1. If $a_{n}^{p} \ell_{n} \rightarrow 0$, then $a_{n} / b_{n} \rightarrow 0, b_{n}^{p} \ell_{n} \rightarrow 0,\left(b_{n}^{p} \ell_{n}\right)^{4 /(p \alpha)} \times$ $\left(b_{n} / a_{n}\right) \rightarrow 0$, and for any $\varepsilon>0$ there is an $N_{\varepsilon}$ such that if $n>N_{\varepsilon}$, then

$$
2^{-2 / \alpha}\left(a_{n}^{p} \ell_{n}\right)^{\varepsilon /(p \alpha)} \leqslant \frac{L_{1}\left(\ell_{n}^{1 / p}\right)}{L_{1}\left(1 / a_{n}\right)} \leqslant 2^{2 /(\alpha p)}\left(a_{n}^{p} \ell_{n}\right)^{-\varepsilon /(2 p)} .
$$


P r o o f. Estimate (21) follows by the Potter bounds (Theorem 1.5.6 in [6]). If $\varepsilon \in(0,2-\alpha)$, then for large enough $n(21)$ implies that

$$
\begin{aligned}
\frac{a_{n}}{b_{n}} & =\frac{a_{n} n^{1 / 2} \ell_{n}^{(2-\alpha) /(2 p)}}{\left[L_{1}\left(\ell_{n}^{1 / p}\right)\right]^{\alpha / 2}}=\frac{\left(a_{n}^{p} \ell_{n}\right)^{(2-\alpha) /(2 p)} a_{n}^{\alpha / 2} n^{1 / 2}}{\left[L_{1}\left(\ell_{n}^{1 / p}\right)\right]^{\alpha / 2}} \\
& =\frac{\left(a_{n}^{p} \ell_{n}\right)^{(2-\alpha) /(2 p)}}{\left[L_{1}\left(\ell_{n}^{1 / p}\right) L_{1}^{\#}\left(n^{1 / \alpha}\right)\right]^{\alpha / 2}} \leqslant \frac{2\left(a_{n}^{p} \ell_{n}\right)^{(2-\alpha-\varepsilon) /(2 p)}}{\left[L_{1}\left(1 / a_{n}\right) L_{1}^{\#}\left(n^{1 / \alpha}\right)\right]^{\alpha / 2}} \rightarrow 0 .
\end{aligned}
$$

Similarly, if we take $\varepsilon=1$ then for large enough $n$ (21) implies that

$$
\begin{aligned}
b_{n}^{p} \ell_{n} & =\left[n^{-p / \alpha} \ell_{n}\right]^{\alpha / 2}\left[L_{1}\left(\ell_{n}^{1 / p}\right)\right]^{\alpha p / 2} \\
& =\left[a_{n}^{p} \ell_{n}\right]^{\alpha / 2}\left(L_{1}^{\#}\left(n^{1 / \alpha}\right) L_{1}\left(\ell_{n}^{1 / p}\right)\right)^{\alpha p / 2} \\
& \leqslant 2\left[a_{n}^{p} \ell_{n}\right]^{\alpha / 4}\left(L_{1}^{\#}\left(n^{1 / \alpha}\right) L_{1}\left(\frac{1}{a_{n}}\right)\right)^{\alpha p / 2} \rightarrow 0
\end{aligned}
$$

and if we take $\varepsilon \in(0, p]$, then for large enough $n(21)$ implies that

$$
\begin{aligned}
\left(b_{n}^{p} \ell_{n}\right)^{4 /(p \alpha)} \frac{b_{n}}{a_{n}} & =\left[a_{n} \ell_{n}^{1 / p}\right]^{2}\left[L_{1}^{\#}\left(n^{1 / \alpha}\right) L_{1}\left(\ell_{n}^{1 / p}\right)\right]^{2} \frac{b_{n}}{a_{n}} \\
& =\left(b_{n} \ell_{n}^{1 / p}\right)\left(a_{n} \ell_{n}^{1 / p}\right)\left[L_{1}^{\#}\left(n^{1 / \alpha}\right) L_{1}\left(\ell_{n}^{1 / p}\right)\right]^{2} \\
& \leqslant 2^{4 /(p \alpha)}\left(b_{n} \ell_{n}^{1 / p}\right)\left[L_{1}^{\#}\left(n^{1 / \alpha}\right) L_{1}\left(\frac{1}{a_{n}}\right)\right]^{2} \rightarrow 0,
\end{aligned}
$$

where the convergence follows by (17). Lemma 1 is proved.

Pro of of $\mathrm{Th}$ e or e m 3. If $S_{n}\left(\ell_{n}\right)$ is given by $(9)$ and $\eta>0$ is a constant, then $\eta S_{n}\left(\ell_{n}\right) \sim \operatorname{ID}\left(0, M_{n}^{\eta}, \gamma_{n}^{\eta}\right)$, where

$$
M_{n}^{\eta}(B)=n \int_{0}^{\infty} 1_{B}(\eta x) e^{-x^{p} / \ell_{n}} M(\mathrm{~d} x), \quad B \in \mathscr{B}(\mathbf{R}),
$$

and

$$
\gamma_{n}^{\eta}=n \eta\left(1-\eta^{2}\right) \int_{0}^{\infty} \frac{x^{3}}{\left(1+x^{2}\right)\left(1+x^{2} \eta^{2}\right)} e^{-x^{p} / \ell_{n}} M(\mathrm{~d} x) .
$$

Case $a_{n}^{p} \ell_{n} \rightarrow c \in(0, \infty]$. By Proposition 1 it suffices to show:

(a) $M_{n}^{a_{n}}(\mathrm{~d} x) \stackrel{v}{\rightarrow} e^{-x^{p} / c} M_{\infty}(\mathrm{d} x)$,

(b) $\lim _{\varepsilon \downarrow 0} \lim _{n \rightarrow \infty} \int_{0}^{\varepsilon} x^{2} M_{n}^{a_{n}}(\mathrm{~d} x)=0$, and

(c) $\gamma_{n}^{a_{n}}-\kappa_{n} \rightarrow 0$.

To show a) let $f$ be any nonnegative bounded continuous function vanishing on a neighborhood of 0 . Note that $f(x) e^{-x^{p} / c}$ is also such a function, thus (20) implies that

$$
\lim _{n \rightarrow \infty} \int_{0}^{\infty} f(x) e^{-x^{p} / c} M_{n}(\mathrm{~d} x)=\int_{0}^{\infty} f(x) e^{-x^{p} / c} M_{\infty}(\mathrm{d} x),
$$


where $M_{n}$ is given by (19). Fix $\varepsilon>0$. There is a point $x_{0}$ such that for all $n$ large enough

$$
\int_{x_{0}}^{\infty} f(x) M_{n}(\mathrm{~d} x)<\frac{\varepsilon}{4} .
$$

Thus for large enough $n$

$$
\begin{aligned}
\int_{0}^{\infty} & f(x)\left|e^{-x^{p} /\left(a_{n}^{p} \ell_{n}\right)}-e^{-x^{p} / c}\right| M_{n}(\mathrm{~d} x) \\
= & \int_{0}^{x_{0}} f(x)\left|e^{-x^{p} /\left(a_{n}^{p} \ell_{n}\right)}-e^{-x^{p} / c}\right| M_{n}(\mathrm{~d} x) \\
& +\int_{x_{0}}^{\infty} f(x)\left|e^{-x^{p} /\left(a_{n}^{p} \ell_{n}\right)}-e^{-x^{p} / c}\right| M_{n}(\mathrm{~d} x) \\
\leqslant & \int_{0}^{x_{0}} f(x)\left|e^{x^{p}\left[1 / c-1 /\left(a_{n}^{p} \ell_{n}\right)\right]}-1\right| M_{n}(\mathrm{~d} x)+\frac{\varepsilon}{2} \\
\leqslant & \left(e^{x_{0}^{p}\left|1 / c-1 /\left(a_{n}^{p} \ell_{n}\right)\right|}-1\right) \int_{0}^{x_{0}} f(x) M_{n}(\mathrm{~d} x)+\frac{\varepsilon}{2}<\varepsilon,
\end{aligned}
$$

where the last line follows by 4.2.39 in [1]. This implies that

$$
\begin{aligned}
\lim _{n \rightarrow \infty}\left|\int_{0}^{\infty} f(x) M_{n}^{a_{n}}(\mathrm{~d} x)-\int_{0}^{\infty} f(x) e^{-x^{p} / c} M_{\infty}(\mathrm{d} x)\right| \\
=\lim _{n \rightarrow \infty}\left|n \int_{0}^{\infty} f\left(x a_{n}\right) e^{-x^{p} / \ell_{n}} M(\mathrm{~d} x)-\int_{0}^{\infty} f(x) e^{-x^{p} / c} M_{\infty}(\mathrm{d} x)\right| \\
=\lim _{n \rightarrow \infty}\left|\int_{0}^{\infty} f(x) e^{-x^{p} /\left(a_{n}^{p} \ell_{n}\right)} M_{n}(\mathrm{~d} x)-\int_{0}^{\infty} f(x) e^{-x^{p} / c} M_{\infty}(\mathrm{d} x)\right| \\
\leqslant \lim _{n \rightarrow \infty} \int_{0}^{\infty} f(x)\left|e^{-x^{p} /\left(a_{n}^{p} \ell_{n}\right)}-e^{-x^{p} / c}\right| M_{n}(\mathrm{~d} x) \\
\quad+\lim _{n \rightarrow \infty}\left|\int_{0}^{\infty} f(x) e^{-x^{p} / c} M_{n}(\mathrm{~d} x)-\int_{0}^{\infty} f(x) e^{-x^{p} / c} M_{\infty}(\mathrm{d} x)\right|=0 .
\end{aligned}
$$

To show (b) note that, by (20),

$$
\begin{aligned}
\lim _{\varepsilon \downarrow 0} & \lim _{n \rightarrow \infty} \int_{0}^{\varepsilon} x^{2} M_{n}^{a_{n}}(\mathrm{~d} x)=\lim _{\varepsilon \downarrow 0} \lim _{n \rightarrow \infty} n a_{n}^{2} \int_{0}^{\varepsilon / a_{n}} x^{2} e^{-x^{p} / \ell_{n}} M(\mathrm{~d} x) \\
& =\lim _{\varepsilon \downarrow 0} \lim _{n \rightarrow \infty} \int_{0}^{\varepsilon} x^{2} e^{-x^{p} /\left(a_{n}^{p} \ell_{n}\right)} M_{n}(\mathrm{~d} x) \leqslant \lim _{\varepsilon \downarrow 0} \lim _{n \rightarrow \infty} \int_{0}^{\varepsilon} x^{2} M_{n}(\mathrm{~d} x)=0 .
\end{aligned}
$$

To show (c) note that

$$
\begin{aligned}
\left|\gamma_{n}^{a_{n}}-\kappa_{n}\right| & =n a_{n}^{3} \int_{0}^{\infty} \frac{x^{3}}{\left(1+x^{2}\right)\left(1+x^{2} a_{n}^{2}\right)} e^{-x^{p} / \ell_{n}} M(\mathrm{~d} x) \\
& \leqslant n a_{n}^{2} \int_{0}^{\infty} \frac{x^{2}}{1+x^{2}} M(\mathrm{~d} x) \rightarrow 0,
\end{aligned}
$$

where the inequality follows from the fact that $x a_{n} /\left(1+x^{2} a_{n}^{2}\right) \leqslant 1$. 
Case $a_{n}^{p} \ell_{n} \rightarrow 0$. It suffices to show:

$\left(\mathrm{a}^{\prime}\right) M_{n}^{b_{n}} \stackrel{v}{\rightarrow} 0$,

$\left(\mathrm{b}^{\prime}\right) \lim _{\varepsilon \downarrow 0} \lim _{n \rightarrow \infty} \int_{0}^{\varepsilon} x^{2} M_{n}^{b_{n}}(\mathrm{~d} x)=(\alpha / p) \Gamma((2-\alpha) / p)$, and

$\left(\mathrm{c}^{\prime}\right) \gamma_{n}^{b_{n}}-\kappa_{n}^{\prime} \rightarrow 0$.

To show $\left(\mathrm{a}^{\prime}\right)$, let $f$ be any nonnegative bounded continuous function vanishing on a neighborhood of 0 . Let $C$ be the bound on $f$ and let $\delta>0$ be such that $f(x)=0$ whenever $|x|<\delta$. For any $\varepsilon>0$

$$
\begin{aligned}
\int_{0}^{\infty} f(x) M_{n}^{b_{n}}(\mathrm{~d} x) & =n \int_{\delta / b_{n}}^{\infty} f\left(x b_{n}\right) e^{-x^{p} / \ell_{n}} M(\mathrm{~d} x) \\
& =\int_{\delta a_{n} / b_{n}}^{\infty} f\left(\frac{x b_{n}}{a_{n}}\right) e^{-x^{p} /\left(a_{n}^{p} \ell_{n}\right)} M_{n}(\mathrm{~d} x) \\
& \leqslant C \int_{\delta a_{n} / b_{n}}^{\varepsilon} e^{-x^{p} /\left(a_{n}^{p} \ell_{n}\right)} M_{n}(\mathrm{~d} x)+C \int_{\varepsilon}^{\infty} e^{-x^{p} /\left(a_{n}^{p} \ell_{n}\right)} M_{n}(\mathrm{~d} x) .
\end{aligned}
$$

By the Portmanteau theorem and (20)

$$
\begin{aligned}
\lim _{\varepsilon \downarrow 0} \lim _{n \rightarrow \infty} \int_{\varepsilon}^{\infty} e^{-x^{p} /\left(a_{n}^{p} \ell_{n}\right)} M_{n}(\mathrm{~d} x) & \leqslant \lim _{\varepsilon \downarrow 0} \lim _{n \rightarrow \infty} e^{-\varepsilon^{p} /\left(a_{n}^{p} \ell_{n}\right)} M_{n}(\{x: x \geqslant \varepsilon\}) \\
& \leqslant \lim _{\varepsilon \downarrow 0} \lim _{n \rightarrow \infty} e^{-\varepsilon^{p} /\left(a_{n}^{p} \ell_{n}\right)} M_{\infty}(\{x: x \geqslant \varepsilon\})=0 .
\end{aligned}
$$

Lemma 1 and (20) imply that

$$
\begin{aligned}
& \lim _{\varepsilon \downarrow 0} \lim _{n \rightarrow \infty} \int_{\delta a_{n} / b_{n}}^{\varepsilon} e^{-x^{p} /\left(a_{n}^{p} \ell_{n}\right)} M_{n}(\mathrm{~d} x) \\
& \quad \leqslant \lim _{\varepsilon \downarrow 0} \lim _{n \rightarrow \infty} e^{-\delta^{p} /\left(b_{n}^{p} \ell_{n}\right)} \frac{b_{n}^{2}}{a_{n}^{2}} \delta^{-2} \int_{\delta a_{n} / b_{n}}^{\varepsilon} x^{2} M_{n}(\mathrm{~d} x) \\
& \quad \leqslant \lim _{\varepsilon \downarrow 0} \lim _{n \rightarrow \infty}\left(b_{n}^{p} \ell_{n}\right)^{-8 /(p \alpha)} e^{-\delta^{p} /\left(b_{n}^{p} \ell_{n}\right)}\left(b_{n}^{p} \ell_{n}\right)^{8 /(p \alpha)} \frac{b_{n}^{2}}{a_{n}^{2}} \delta^{-2} \int_{0}^{\varepsilon} x^{2} M_{n}(\mathrm{~d} x)=0 .
\end{aligned}
$$

To show $\left(b^{\prime}\right)$ we begin by defining

$$
M_{n}^{\prime}(A)=V\left(\ell_{n}^{1 / p}\right) \int_{0}^{\infty} 1_{A}\left(x \ell_{n}^{-1 / p}\right) M(\mathrm{~d} x), \quad A \in \mathfrak{B}(\mathbf{R}) .
$$

Note that for any $t>0$

$$
\begin{aligned}
\lim _{n \rightarrow \infty} M_{n}^{\prime}(\{x: x>t\}) & =\lim _{n \rightarrow \infty} \frac{M\left(\left\{x: x>t \ell_{n}^{1 / p}\right\}\right)}{M\left(\left\{x: x>\ell_{n}^{1 / p}\right\}\right)} \\
& =t^{-\alpha}=M_{\infty}(\{x: x>t\}),
\end{aligned}
$$

which implies that $M_{n}^{\prime} \stackrel{v}{\rightarrow} M_{\infty}$. We have

$$
\int_{0}^{\varepsilon} x^{2} M_{n}^{b_{n}}(\mathrm{~d} x)=n b_{n}^{2} \int_{0}^{\varepsilon / b_{n}} x^{2} e^{-x^{p} / \ell_{n}} M(\mathrm{~d} x)
$$




$$
\begin{aligned}
& =\ell_{n}^{-2 / p} V\left(\ell_{n}^{1 / p}\right) \int_{0}^{\varepsilon n^{1 / 2} \ell_{n}^{1 / p} / \sqrt{V\left(\ell_{n}^{1 / p}\right)}} x^{2} e^{-x^{p} / \ell_{n}} M(\mathrm{~d} x) \\
& =\int_{0}^{\varepsilon n^{1 / 2} / \sqrt{V\left(\ell_{n}^{1 / p}\right)}} x^{2} e^{-x^{p}} M_{n}^{\prime}(\mathrm{d} x) .
\end{aligned}
$$

We will now show that

$$
\lim _{n \rightarrow \infty} \int_{0}^{\varepsilon n^{1 / 2} / \sqrt{V\left(\ell_{n}^{1 / p}\right)}} x^{2} e^{-x^{p}} M_{n}^{\prime}(\mathrm{d} x)=\frac{\alpha}{p} \Gamma\left(\frac{2-\alpha}{p}\right) .
$$

First, note that $V \in \mathrm{RV}_{\alpha}$, thus by (18) and the Potter bounds (see [6, Theorem 1.5.6]), for any $\gamma \in(0, \alpha)$

$$
\lim _{n \rightarrow \infty} \frac{n}{V\left(\ell_{n}^{1 / p}\right)}=\lim _{n \rightarrow \infty} \frac{V\left(1 / a_{n}\right)}{V\left(\ell_{n}^{1 / p}\right)} \geqslant \lim _{n \rightarrow \infty} 0.5\left(\ell_{n} a_{n}^{p}\right)^{-(\alpha-\gamma) / p}=\infty .
$$

Thus $\varepsilon n^{1 / 2} / \sqrt{V\left(\ell_{n}^{1 / p}\right)} \rightarrow \infty$ and

$$
\begin{aligned}
\lim _{\delta \downarrow 0} \lim _{n \rightarrow \infty} \int_{\delta}^{\varepsilon n^{1 / 2} / \sqrt{V\left(\ell_{n}^{1 / p}\right)}} x^{2} e^{-x^{p}} M_{n}^{\prime}(\mathrm{d} x) & =\int_{0}^{\infty} x^{2} e^{-x^{p}} M_{\infty}(\mathrm{d} x) \\
& =\frac{\alpha}{p} \Gamma\left(\frac{2-\alpha}{p}\right) .
\end{aligned}
$$

By (4), (5), and [16, Section VIII.9, Theorem 2] we have

$$
\begin{aligned}
\lim _{\delta \downarrow 0} \lim _{n \rightarrow \infty} & \int_{0}^{\delta} x^{2} e^{-x^{p}} M_{n}^{\prime}(\mathrm{d} x) \leqslant \lim _{\delta \downarrow 0} \lim _{n \rightarrow \infty} \ell_{n}^{-2 / p} \frac{\int_{0}^{\delta \ell_{n}^{1 / p}} x^{2} M(\mathrm{~d} x)}{M\left(\left\{x: x>\ell_{n}^{1 / p}\right\}\right)} \\
& =\lim _{\delta \downarrow 0} \lim _{n \rightarrow \infty} \delta^{2} \frac{\int_{0}^{\delta \ell_{n}^{1 / p}} x^{2} M(\mathrm{~d} x)}{\left(\delta \ell_{n}^{1 / p}\right)^{2} M\left(\left\{x: x>\delta \ell_{n}^{1 / p}\right\}\right)} \frac{M\left(\left\{x: x>\delta \ell_{n}^{1 / p}\right\}\right)}{M\left(\left\{x: x>\ell_{n}^{1 / p}\right\}\right)} \\
& =\lim _{\delta \downarrow 0} \delta^{2-\alpha} \frac{\alpha}{2-\alpha}=0 .
\end{aligned}
$$

We can show that $\left(c^{\prime}\right)$ holds by arguments similar to the previous case. Theorem 3 is proved.

Proof of $\mathrm{Th}$ e or e m 4. Fix $\alpha \in(1,2)$. If $a_{n}^{p} \ell_{n} \rightarrow c \in(0, \infty]$, then

$$
\begin{aligned}
\frac{S_{n}\left(\ell_{n}\right)}{n} & =\frac{a_{n} S_{n}\left(\ell_{n}\right)-\kappa_{n}}{n a_{n}}+\frac{\kappa_{n}}{n a_{n}} \stackrel{d}{\rightarrow} \lim _{n \rightarrow \infty} \frac{\kappa_{n}}{n a_{n}} \\
& =\lim _{n \rightarrow \infty} \int_{0}^{\infty} \frac{x^{3}}{1+x^{2}} \frac{1}{1+x^{2} a_{n}^{2}} e^{-x^{p} / \ell_{n}} M(\mathrm{~d} x)=\int_{0}^{\infty} \frac{x^{3}}{1+x^{2}} M(\mathrm{~d} x),
\end{aligned}
$$

where the convergence in distribution follows by Theorem 3, Slutsky's theorem, and the fact that $n a_{n} \rightarrow \infty$, and the final convergence follow by 
Lebesgue's dominated convergence theorem. If $a_{n}^{p} \ell_{n} \rightarrow 0$ the proof is similar, we just need to show that $n b_{n} \rightarrow \infty$. To see this note that for any $\varepsilon \in(0,2 \alpha-2)$

$$
n b_{n}=n^{1 / 2} a_{n}^{(2-\alpha+\varepsilon) / 2}\left(a_{n}^{p} \ell_{n}\right)^{-(2-\alpha+\varepsilon) /(2 p)} \ell_{n}^{\varepsilon /(2 p)}\left[L\left(\ell_{n}^{1 / p}\right)\right]^{-1 / 2} \rightarrow \infty .
$$

Fix $\alpha \in(0,1)$. When $a_{n}^{p} \ell_{n} \rightarrow c \in(0, \infty]$

$$
a_{n}^{\prime} S_{n}\left(\ell_{n}\right)=\frac{a_{n}^{\prime}}{a_{n}}\left(a_{n} S_{n}\left(\ell_{n}\right)-\kappa_{n}\right)+\frac{a_{n}^{\prime}}{a_{n}} \kappa_{n} .
$$

By Theorem 3 and Slutsky's theorem it suffices to show that lim sup $\kappa_{n}<\infty$. To see this note that

$$
\begin{aligned}
& \limsup _{n \rightarrow \infty} \kappa_{n}=\limsup _{n \rightarrow \infty} n a_{n} \int_{0}^{\infty} \frac{x^{3}}{\left(1+x^{2}\right)\left(1+x^{2} a_{n}^{2}\right)} e^{-x^{p} / \ell_{n}} M(\mathrm{~d} x) \\
& \quad \leqslant \lim _{n \rightarrow \infty}\left(n a_{n} \int_{0}^{1} x^{2} M(\mathrm{~d} x)+n a_{n} \int_{1}^{1 / a_{n}} x M(\mathrm{~d} x)+n M\left(\left\{x: x \geqslant 1 / a_{n}\right\}\right)\right) \\
& \quad=\lim _{n \rightarrow \infty} \frac{\int_{1}^{1 / a_{n}} x M(\mathrm{~d} x)}{a_{n}^{-1} M\left(\left\{x: x>1 / a_{n}\right\}\right)}+1=\frac{\alpha}{1-\alpha}+1<\infty,
\end{aligned}
$$

where third line follows by (18) and the final equality follows by [16, Section VIII.9, Theorem 2]. When $a_{n}^{p} \ell_{n} \rightarrow 0$

$$
\begin{aligned}
\kappa_{n}^{\prime} & \geqslant n b_{n} \frac{e^{-1}}{2} \frac{1}{1+\left(\ell_{n} b_{n}^{p}\right)^{2 / p}} \int_{1}^{\ell_{n}^{1 / p}} x M(\mathrm{~d} x) \\
& \sim C n^{1 / 2} \ell_{n}^{-1 / p+\alpha /(2 p)}\left[L\left(\ell^{1 / p}\right)\right]^{-1 / 2} \ell_{n}^{(1-\alpha) / p} L\left(\ell^{1 / p}\right) \\
& =C\left(a_{n}^{p} \ell_{n}\right)^{-\alpha /(2 p)}\left[L_{1}^{\#}\left(n^{1 / \alpha}\right) L_{1}\left(\ell^{1 / p}\right)\right]^{-\alpha / 2} \\
& \geqslant C \cdot 2^{-1 / p}\left(a_{n}^{p} \ell_{n}\right)^{-\alpha /(4 p)}\left[L_{1}^{\#}\left(n^{1 / \alpha}\right) L_{1}\left(\frac{1}{a_{n}}\right)\right]^{-\alpha / 2} \rightarrow \infty,
\end{aligned}
$$

where $C>0$ is a constant, the second line follows by Lemma 1 and [16, Section VIII.9, Theorem 2], and the last line follows by (21) and (17). From here the result follows by Theorem 3 and Slutsky's theorem.

Now assume that $\alpha=1$. We will show that $\kappa_{n} \rightarrow \infty$ (or $\kappa_{n}^{\prime} \rightarrow \infty$ when appropriate). From this, the result will follow as in the previous case. First consider the case $\int_{1}^{\infty} x M(\mathrm{~d} x)<\infty$. By Markov's inequality $M(\{x: x>$ $t\}) \leqslant t^{-1} \int_{t}^{\infty} x M(\mathrm{~d} x)$, thus $L(t) \leqslant \int_{t}^{\infty} x M(\mathrm{~d} x) \rightarrow 0$ as $t \rightarrow \infty$. This implies that $\lim _{t \rightarrow \infty} L_{1}(t)=\infty$ and $\lim _{t \rightarrow \infty} L_{1}^{\#}(t)=0$. If $a_{n}^{p} \ell_{n} \rightarrow 0$, then

$$
\begin{aligned}
n b_{n} & =n^{1 / 2} \ell_{n}^{-1 /(2 p)}\left[L\left(\ell_{n}^{1 / p}\right)\right]^{-1 / 2}=\left(a_{n} n\right)^{1 / 2}\left(a_{n}^{p} \ell_{n}\right)^{-1 /(2 p)}\left[L_{1}\left(\ell_{n}^{1 / p}\right)\right]^{1 / 2} \\
& =\left(a_{n}^{p} \ell_{n}\right)^{-1 /(2 p)}\left(\frac{L_{1}\left(\ell_{n}^{1 / p}\right)}{L_{1}^{\#}(n)}\right)^{1 / 2} \rightarrow \infty,
\end{aligned}
$$


which implies that $\kappa_{n}^{\prime} \rightarrow \infty$. If $a_{n}^{p} \ell_{n} \rightarrow c \in(0, \infty]$, then

$$
\begin{aligned}
\kappa_{n} & =n a_{n} \int_{0}^{\infty} \frac{x^{3}}{1+x^{2}} \frac{1}{1+x^{2} a_{n}^{2}} e^{-x^{p} / \ell_{n}} M(\mathrm{~d} x) \\
& \sim \frac{1}{L_{1}^{\#}(n)} \int_{0}^{\infty} \frac{x^{3}}{1+x^{2}} M(\mathrm{~d} x) \rightarrow \infty .
\end{aligned}
$$

Now consider the case $\int_{1}^{\infty} x M(\mathrm{~d} x)=\infty$. If $a_{n}^{p} \ell_{n} \rightarrow 0$, then by Lemma 1

$$
\begin{aligned}
\kappa_{n}^{\prime} & \geqslant n b_{n} \int_{1}^{\ell_{n}^{1 / p}} x M(\mathrm{~d} x) \frac{0.5 e^{-1}}{1+\left(b_{n}^{p} \ell_{n}\right)^{2 / p}} \\
& \sim C\left(a_{n} n\right)^{1 / 2}\left(a_{n}^{p} \ell_{n}\right)^{-1 /(2 p)}\left[L\left(\ell_{n}^{1 / p}\right)\right]^{1 / 2} \frac{\int_{1}^{\ell_{n}^{1 / p}} x M(\mathrm{~d} x)}{L\left(\ell_{n}^{1 / p}\right)} \\
& =C\left(a_{n}^{p} \ell_{n}\right)^{-1 /(2 p)}\left[L_{1}\left(\ell_{n}^{1 / p}\right) L_{1}^{\#}(n)\right]^{-1 / 2} \frac{\int_{1}^{\ell_{n}^{1 / p}} x M(\mathrm{~d} x)}{L\left(\ell_{n}^{1 / p}\right)} \\
& \geqslant C \cdot 2^{-1 / p}\left(a_{n}^{p} \ell_{n}\right)^{-1 /(4 p)}\left[L_{1}\left(\frac{1}{a_{n}}\right) L_{1}^{\#}(n)\right]^{-1 / 2} \frac{\int_{1}^{\ell_{n}^{1 / p}} x M(\mathrm{~d} x)}{\ell_{n}^{1 / p} M\left(\left\{x: x>\ell_{n}^{1 / p}\right\}\right)} \rightarrow \infty,
\end{aligned}
$$

where $C=0.5 e^{-1}$ and the convergence follows by (17) and [16, Section VIII.9, Theorem 2]. Similarly, if $a_{n}^{p} \ell_{n} \rightarrow c \in(0, \infty]$, then for some $K>0$ and large enough $n$

$$
\kappa_{n} \geqslant \frac{K}{L_{1}^{\#}(n)} \int_{1}^{1 / a_{n}} x M(\mathrm{~d} x)=\frac{K}{L_{1}^{\#}(n) L_{1}\left(1 / a_{n}\right)} \frac{\int_{1}^{1 / a_{n}} x M(\mathrm{~d} x)}{a_{n}^{-1} M\left(\left\{x: x>1 / a_{n}\right\}\right)},
$$

which converges to infinity by [16, Section VIII.9, Theorem 2] and (17). Theorem 4 is proved.

6. Proofs for subsection 2.3. We begin by stating, in the context of our situation, several well-known facts relating convergence of sequences of infinitely divisible distributions to convergence of sums of infinitesimal triangular arrays. These facts follow from, e.g., [29, Theorem 3.2.2] and Proposition 1.

Let $X_{1}\left(\ell_{n}\right), X_{2}\left(\ell_{n}\right), \ldots, X_{n}\left(\ell_{n}\right) \stackrel{\text { iid }}{\sim} \mu_{p}^{\left(\ell_{n}\right)}$ and let $Y_{1}\left(\ell_{n}\right), Y_{2}\left(\ell_{n}\right), \ldots$, $Y_{n}\left(\ell_{n}\right) \stackrel{\mathrm{iid}}{\sim} \operatorname{ID}\left(0, \mu_{p}^{\left(\ell_{n}\right)}, 0\right)$. Assume that $\left\{\gamma_{n}\right\}$ is a sequence of positive numbers such that $\gamma_{n} X_{1}\left(\ell_{n}\right) \stackrel{p}{\rightarrow} 0$ and

$$
\gamma_{n} \sum_{i=1}^{n} Y_{i}\left(\ell_{n}\right)-\gamma_{n} n\left(1-\gamma_{n}^{2}\right) \int_{0}^{\infty} \frac{x^{3}}{\left(1+x^{2}\right)\left(1+x^{2} \gamma_{n}^{2}\right)} \mu_{p}^{\left(\ell_{n}\right)}(\mathrm{d} x) \stackrel{d}{\rightarrow} Y,
$$

where $Y \sim \operatorname{ID}(a, M, 0)$. If $a=0, M \neq 0$, and $M(\{x:|x|=1\})=0$, then

$$
\gamma_{n} \sum_{i=1}^{n} X_{i}\left(\ell_{n}\right)-\gamma_{n} n \int_{0}^{1 / \gamma_{n}} x \mu_{p}^{\left(\ell_{n}\right)}(\mathrm{d} x) \stackrel{d}{\rightarrow} Z,
$$


where $Z \sim \operatorname{ID}(0, M, b)$ and

$$
b=\int_{0}^{\infty} x\left(\frac{1}{1+x^{2}}-1_{\{x<1\}}\right) M(\mathrm{~d} x) .
$$

If $a>0, M=0$, and

$$
\lim _{\varepsilon \rightarrow 0} \limsup _{n \rightarrow \infty} \sqrt{n} \gamma_{n} \int_{0}^{\varepsilon / \gamma_{n}} x \mu_{p}^{\left(\ell_{n}\right)}(\mathrm{d} x)=0,
$$

then (25) holds with $Z \sim N(0, a)$.

Assume further that $\gamma_{n}^{2} n \rightarrow 0$ and let $Y_{1}^{\prime}\left(\ell_{n}\right), Y_{2}^{\prime}\left(\ell_{n}\right), \ldots, Y_{n}^{\prime}\left(\ell_{n}\right) \stackrel{\text { iid }}{\sim}$ ID $\left(0, \mu_{n}^{\prime}, 0\right)$, where $\mu_{n}^{\prime}(\mathrm{d} x)=c_{\ell_{n}}^{-1} \mu_{p}^{\left(\ell_{n}\right)}(\mathrm{d} x)=e^{-x / \ell_{n}} \mu(\mathrm{d} x)$. By Proposition 1 , the fact that $c_{\ell_{n}} \rightarrow 1$, and (22) it follows that (24) holds if and only if

$$
\gamma_{n} \sum_{i=1}^{n} Y_{i}^{\prime}\left(\ell_{n}\right)-\gamma_{n} n \int_{0}^{\infty} \frac{x^{3}}{\left(1+x^{2}\right)\left(1+x^{2} \gamma_{n}^{2}\right)} e^{-x^{p} / \ell_{n}} \mu(\mathrm{d} x) \stackrel{d}{\rightarrow} Y .
$$

We will now use these facts to prove Theorem 5 . If $a_{n}^{p} \ell_{n} \rightarrow c \in(0, \infty]$, then Theorem 3 implies that (26) holds with $\gamma_{n}=a_{n}$ for $n=1,2, \ldots$ and $Y=R_{\alpha, c}$, which gives the result.

Now assume that $a_{n}^{p} \ell_{n} \rightarrow 0$. By Theorem 3 this implies that (26) holds with $\gamma_{n}=b_{n}$ for $n=1,2, \ldots$ and $Y \sim N\left(0, \sigma^{2}\right)$, where $\sigma^{2}$ is as in Theorem 5 . When $\alpha \in(1,2)$ we have

$$
\sqrt{n} b_{n} \int_{0}^{\varepsilon / b_{n}} x \mu_{p}^{\left(\ell_{n}\right)}(\mathrm{d} x) \leqslant c_{\ell_{n}} \ell_{n}^{-(2-\alpha) /(2 p)}\left[L\left(\ell_{n}^{1 / p}\right)\right]^{-1 / 2} \int_{0}^{\infty} x \mu(\mathrm{d} x) \rightarrow 0 .
$$

Now assume that $\alpha \in(0,1]$. Note that

$$
\begin{aligned}
& \sqrt{n} b_{n} \int_{0}^{\varepsilon / b_{n}} x \mu_{p}^{\left(\ell_{n}\right)}(\mathrm{d} x) \leqslant c_{\ell_{n}} \sqrt{n} b_{n}\left(\int_{0}^{\ell_{n}^{1 / p}} x \mu(\mathrm{d} x)+\int_{\ell_{n}^{1 / p}}^{\infty} x e^{-x^{p} / \ell_{n}} \mu(\mathrm{d} x)\right) \\
&=: I_{1, n}+I_{2, n} .
\end{aligned}
$$

Fix $\varepsilon^{\prime} \in(0, \alpha / 2)$ and note that

$$
\begin{aligned}
\limsup _{n \rightarrow \infty} I_{1, n} & \leqslant \lim _{n \rightarrow \infty} \sqrt{n} b_{n}\left(\int_{0}^{1} x \mu(\mathrm{d} x)+\int_{0}^{\ell_{n}^{1 / p}} x^{1+\varepsilon^{\prime}} \mu(\mathrm{d} x)\right) \\
& =\lim _{n \rightarrow \infty} \sqrt{n} b_{n} \int_{0}^{\ell_{n}^{1 / p}} x^{1+\varepsilon^{\prime}} \mu(\mathrm{d} x) \\
& =\lim _{n \rightarrow \infty} \frac{1}{\sqrt{V\left(\ell_{n}^{1 / p}\right)}} \frac{\int_{0}^{\ell_{n}^{1 / p}} x^{1+\varepsilon^{\prime}} \mu(\mathrm{d} x)}{\ell_{n}^{1 / p} \mu\left(\left\{x: x>\ell^{1 / p}\right\}\right)} \\
& =\lim _{n \rightarrow \infty} \frac{\sqrt{L\left(\ell_{n}^{1 / p}\right)}}{\ell_{n}^{\left(\alpha / 2-\varepsilon^{\prime}\right) / p}} \frac{\int_{0}^{\ell_{n}^{1 / p}} x^{1+\varepsilon^{\prime}} \mu(\mathrm{d} x)}{\ell_{n}^{\left(1+\varepsilon^{\prime}\right) / p} \mu\left(\left\{x: x>\ell^{1 / p}\right\}\right)} \\
& =\lim _{n \rightarrow \infty} \sqrt{L\left(\ell_{n}^{1 / p}\right)} \ell_{n}^{-\left(\alpha / 2-\varepsilon^{\prime}\right) / p} \frac{\alpha}{1+\varepsilon^{\prime}-\alpha}=0,
\end{aligned}
$$


where the second line follows by the fact that $\sqrt{n} b_{n} \rightarrow 0$ and the fifth by [16, Section VIII.9, Theorem 2]. Now note that

$$
I_{2, n}=c_{\ell_{n}} \ell_{n}^{-1 / p} \sqrt{V\left(\ell_{n}^{1 / p}\right)} \int_{\ell_{n}^{1 / p}}^{\infty} x e^{-x^{p} / \ell_{n}} \mu(\mathrm{d} x)=c_{\ell_{n}} \int_{1}^{\infty} x e^{-x^{p}} M_{n}^{\prime \prime}(\mathrm{d} x),
$$

where

$$
M_{n}^{\prime \prime}(A)=\sqrt{V\left(\ell_{n}^{1 / p}\right)} \int_{0}^{\infty} 1_{A}\left(x \ell_{n}^{-1 / p}\right) \mu(\mathrm{d} x), \quad A \in \mathfrak{B}(\mathbf{R})
$$

By arguments similar to those in (23) we have $M_{n}^{\prime \prime} \stackrel{v}{\rightarrow} 0$. Hence, by the Portmanteau theorem, $\lim _{n \rightarrow \infty} I_{2, n}=0$. This completes the proof of Theorem 5 .

The proof of Theorem 6 is similar to that of Theorem 4 and is thus omitted.

7. Proofs for Section 3. We begin with several lemmas.

Lemma 2. If $F_{1}$ belongs to the domain of attraction of the Gaussian, then there is an $L \in R V_{0}$ such that

$$
\lim _{n \rightarrow \infty} L(n)<\infty \quad \text { and } \quad c_{1}(n)=n^{-1 / 2} L(n) .
$$

$\mathrm{P}$ r o o f. If $F_{1}$ has a finite second moment, then we can take $c_{1}(n)=$ $n^{-1 / 2}$ and the result follows. Henceforth, assume that the second moment is infinite. Let $U(t)=\int_{-t}^{t} x^{2} \mathrm{~d} F_{1}(x), L_{1}(t)=[U(t)]^{-1 / 2}$, and let $V(t)=t^{2}\left[L_{1}(t)\right]^{2}$. Note that $U(t) \rightarrow \infty$ as $t \rightarrow \infty$. By the results in $[16$, Section XVII.5], the assumptions imply that $U \in \mathrm{RV}_{0}$ and that we can take $c_{1}(n)=1 / V^{\leftarrow}(n)$, where $V^{\leftarrow}$ is defined as in (6). This implies that there is an $L \in \mathrm{RV}_{0}$ such that $c_{1}(n)=n^{-1 / 2} L(n)$. If $L_{1}^{\#}(t)=1 / L\left(t^{2}\right)$, then by $[6$, Theorem 1.5.15]

$$
\lim _{n \rightarrow \infty}[L(n)]^{-1} L_{1}\left(n^{1 / 2} L_{1}^{\#}\left(n^{1 / 2}\right)\right)=\lim _{n \rightarrow \infty} L_{1}^{\#}\left(n^{1 / 2}\right) L_{1}\left(n^{1 / 2} L_{1}^{\#}\left(n^{1 / 2}\right)\right)=1 .
$$

Since $L_{1}\left(n^{1 / 2} L_{1}^{\#}\left(n^{1 / 2}\right)\right) \rightarrow 0$, it follows that $L(n) \rightarrow 0$. Lemma 2 is proved.

Lemma 3. Assume that $N_{n} \sim B\left(n, p_{n}\right)$ and let $A_{i}$ be any sequence of random variables. If $n p_{n} \rightarrow 0$, then $\sum_{i=1}^{N_{n}} A_{i} \stackrel{p}{\rightarrow} 0$.

P r o o f. The fact that

$$
\begin{aligned}
\mathbf{P}\left(\left|\sum_{i=1}^{N_{n}} A_{i}\right|>\varepsilon\right) & \leqslant \mathbf{P}\left(N_{n}>0\right)=1-\mathbf{P}\left(N_{n}=0\right) \\
& =1-\left(1-p_{n}\right)^{n}=1-\exp \left\{n p_{n} \frac{\ln \left(1-p_{n}\right)}{p_{n}}\right\} \rightarrow 0
\end{aligned}
$$

proves the result. Lemma 3 is proved. 
Lemma 4. Fix $\gamma \in(0,2]$ and let $g \in \mathrm{RV}_{-1 / \gamma}$. If $\gamma=2$ assume that

$$
\limsup _{t \rightarrow \infty} t^{1 / 2} g(t)<\infty \text {. }
$$

If $N_{n} \sim B\left(n, p_{n}\right), p_{n} \rightarrow 0$, and $n p_{n} \rightarrow \infty$, then $N_{n} \stackrel{p}{\rightarrow} \infty, N_{n} /\left(n p_{n}\right) \stackrel{p}{\rightarrow} 1$, $g\left(N_{n}\right) / g(n p) \stackrel{p}{\rightarrow} 1, g(n)\left(N_{n}-n p_{n}\right) \stackrel{p}{\rightarrow} 0$, and if $\gamma \neq 2$, then $g\left(n p_{n}\right)\left(N_{n}-\right.$ $\left.n p_{n}\right) \stackrel{p}{\rightarrow} 0$.

P r o of. The fact that $\mathbf{E}\left[N_{n} /\left(n p_{n}\right)\right]=1$ and $\mathbf{D}\left(N_{n} /\left(n p_{n}\right)\right)=$ $\left(1-p_{n}\right) /\left(n p_{n}\right) \rightarrow 0$ implies that $N_{n} /\left(n p_{n}\right) \stackrel{p}{\rightarrow} 1$. This and the results in [30, p. 36] imply that $g\left(N_{n}\right) / g\left(n p_{n}\right) \stackrel{p}{\rightarrow} 1$. The facts that

$$
\mathbf{P}\left(g(n)\left|N_{n}-n p_{n}\right|>\varepsilon\right) \leqslant[g(n)]^{2} \frac{\mathbf{D}\left(N_{n}\right)}{\varepsilon^{2}}=\varepsilon^{-2}[g(n)]^{2} n p_{n}\left(1-p_{n}\right) \rightarrow 0
$$

and when $\gamma \neq 2$

$\mathbf{P}\left(g\left(n p_{n}\right)\left|N_{n}-n p_{n}\right|>\varepsilon\right) \leqslant\left[g\left(n p_{n}\right)\right]^{2} \frac{\mathbf{D}\left(N_{n}\right)}{\varepsilon^{2}}=\varepsilon^{-2}\left[g\left(n p_{n}\right)\right]^{2} n p_{n}\left(1-p_{n}\right) \rightarrow 0$

give the last two parts. Lemma 4 is proved.

P r o of of The or e m 7. We will use (13) and (14) throughout without comment. If $n p_{n} \rightarrow 0$, then Lemma 3 and Slutsky's theorem imply that

$$
\begin{aligned}
c_{\alpha_{1}}(n) \sum_{i=1}^{n}\left(Z_{i}^{(n)}-b_{\alpha_{1}}\right) & \stackrel{d}{=} c_{\alpha_{1}}(n) \sum_{i=N_{n}+1}^{n} X_{i}+c_{\alpha_{1}}(n) \sum_{i=1}^{N_{n}} Y_{i}-n c_{\alpha_{1}}(n) b_{\alpha_{1}} \\
& =c_{\alpha_{1}}(n) \sum_{i=1}^{n}\left(X_{i}-b_{\alpha_{1}}\right)+c_{\alpha_{1}}(n) \sum_{i=1}^{N_{n}}\left(Y_{i}-X_{i}\right) \stackrel{d}{\rightarrow} R_{\alpha_{1}} .
\end{aligned}
$$

If $n p_{n} \rightarrow \eta \in(0, \infty)$, then by the compound Poisson approximation of the compound binomial and Slutsky's theorem

$$
c_{\alpha_{1}}(n) \sum_{i=1}^{n}\left(Z_{i}^{(n)}-b_{\alpha_{1}}\right) \stackrel{d}{=} c_{\alpha_{1}}(n) \sum_{i=1}^{n}\left(X_{i}-b_{\alpha_{1}}\right)+c_{\alpha_{1}}(n) \sum_{i=1}^{N_{n}}\left(Y_{i}-X_{i}\right) \stackrel{d}{\rightarrow} R_{\alpha_{1}} .
$$

Now assume that $n p_{n} \rightarrow \infty$. Lemma 4 and Slutsky's theorem imply that

$$
\begin{aligned}
c_{\alpha_{2}}\left(n p_{n}\right) & \sum_{i=1}^{N_{n}}\left(Y_{i}-b_{\alpha_{2}}+b_{\alpha_{1}}-X_{i}\right) \\
= & \frac{c_{\alpha_{2}}\left(n p_{n}\right)}{c_{\alpha_{2}}\left(N_{n}\right)} c_{\alpha_{2}}\left(N_{n}\right) \sum_{i=1}^{N_{n}}\left(Y_{i}-b_{\alpha_{2}}+b_{\alpha_{1}}-X_{i}\right) \stackrel{d}{\rightarrow} R_{\alpha_{2}},
\end{aligned}
$$


where the convergence holds by the fact that $N_{n} \stackrel{p}{\rightarrow} \infty$ and a standard result that, in this case, we can replace $n$ with $N_{n}$ in (13), see, e.g., [23, Theorem 1.1]. If $c_{\alpha_{1}}(n) / c_{\alpha_{2}}\left(p_{n} n\right) \rightarrow 0$, then

$$
\begin{aligned}
& c_{\alpha_{1}}(n) \sum_{i=1}^{n}\left(Z_{i}^{(n)}-b_{\alpha_{1}}-p_{n}\left(b_{\alpha_{2}}-b_{\alpha_{1}}\right)\right) \\
& \quad \stackrel{d}{=} c_{\alpha_{1}}(n) \sum_{i=1}^{n}\left(X_{i}-b_{\alpha_{1}}\right)+\frac{c_{\alpha_{1}}(n)}{c_{\alpha_{2}}\left(p_{n} n\right)} c_{\alpha_{2}}\left(p_{n} n\right) \sum_{i=1}^{N_{n}}\left(Y_{i}-b_{\alpha_{2}}+b_{\alpha_{1}}-X_{i}\right) \\
& \quad+\left(b_{\alpha_{2}}-b_{\alpha_{1}}\right) c_{\alpha_{1}}(n)\left(N_{n}-p_{n} n\right) \stackrel{d}{\rightarrow} R_{\alpha_{1}},
\end{aligned}
$$

where the convergence follows from Lemma 4 (Lemma 2 implies that (27) holds when $\left.\alpha_{1}=2\right)$ and Slutsky's theorem. If $c_{\alpha_{1}}(n) / c_{\alpha_{2}}\left(p_{n} n\right) \rightarrow \kappa \in(0, \infty]$, then

$$
\begin{aligned}
& c_{\alpha_{2}}\left(p_{n} n\right) \sum_{i=1}^{n}\left(Z_{i}^{(n)}-b_{\alpha_{1}}-p_{n}\left(b_{\alpha_{2}}-b_{\alpha_{1}}\right)\right) \\
& \quad \stackrel{d}{=} \frac{c_{\alpha_{2}}\left(n p_{n}\right)}{c_{\alpha_{1}}(n)} c_{\alpha_{1}}(n) \sum_{i=1}^{n}\left(X_{i}-b_{\alpha_{1}}\right)+c_{\alpha_{2}}\left(n p_{n}\right) \sum_{i=1}^{N_{n}}\left(Y_{i}-b_{\alpha_{2}}+b_{\alpha_{1}}-X_{i}\right) \\
& \quad+\left(b_{\alpha_{2}}-b_{\alpha_{1}}\right) c_{\alpha_{2}}\left(n p_{n}\right)\left(N_{n}-n p_{n}\right) \stackrel{d}{\rightarrow} \kappa^{-1} R_{\alpha_{1}}+R_{\alpha_{2}},
\end{aligned}
$$

where the convergence follows by Lemma 4 and Slutsky's theorem. Theorem 7 is proved.

P r o of of The or e m 8. Since $\alpha_{1} \in(1,2]$, we have $\mathbf{E}\left|X_{1}\right|<\infty$. Thus by the law of large numbers and Slutsky's theorem

$$
\begin{aligned}
\lim _{n \rightarrow \infty} \frac{1}{n} \sum_{i=1}^{n} Z_{i}^{(n)} & \stackrel{d}{=} \lim _{n \rightarrow \infty} \frac{1}{n}\left(\sum_{i=1}^{n} X_{i}+\sum_{i=1}^{N_{n}}\left(Y_{i}-X_{i}\right)\right) \\
& \stackrel{d}{=} \mathbf{E}\left[X_{1}\right]+\lim _{n \rightarrow \infty} \frac{1}{n} \sum_{i=1}^{N_{n}}\left(Y_{i}-X_{i}\right) .
\end{aligned}
$$

If $n p_{n} \rightarrow 0$, then the result follows by Lemma 3. If $n p_{n} \rightarrow \eta \in(0, \infty)$, then the compound Poisson approximation of the compound binomial gives the result. If $n p_{n} \rightarrow \infty$ and $n c_{\alpha_{2}}\left(n p_{n}\right) \rightarrow \kappa \in(0, \infty]$, then

$$
\begin{aligned}
\frac{1}{n} \sum_{i=1}^{N_{n}}\left(Y_{i}-X_{i}\right)= & \frac{1}{n c_{\alpha_{2}}\left(n p_{n}\right)} c_{\alpha_{2}}\left(n p_{n}\right) \sum_{i=1}^{N_{n}}\left(Y_{i}-b_{\alpha_{2}}+b_{\alpha_{1}}-X_{i}\right) \\
& +\left(b_{\alpha_{2}}-b_{\alpha_{1}}\right) \frac{N_{n}}{n p_{n}} p_{n} \stackrel{d}{\rightarrow} \kappa^{-1} R_{\alpha_{2}},
\end{aligned}
$$

where the convergence follows from (28), Lemma 4, and Slutsky's theorem. If $n p_{n} \rightarrow \infty$ and $n c_{\alpha_{2}}\left(n p_{n}\right) \rightarrow 0$, then

$$
\lim _{n \rightarrow \infty} \frac{c_{\alpha_{1}}(n)}{c_{\alpha_{2}}\left(n p_{n}\right)}=\lim _{n \rightarrow \infty} \frac{n c_{\alpha_{1}}(n)}{n c_{\alpha_{2}}\left(n p_{n}\right)}=\infty,
$$


since $\alpha_{1} \in(1,2]$. If $\left\{a_{n}\right\}$ satisfies (15), then Theorem 7 implies that

$$
a_{n} \sum_{i=1}^{n} Z_{i}^{(n)}=\frac{a_{n}}{c_{\alpha_{2}}\left(n p_{n}\right)} c_{\alpha_{2}}\left(n p_{n}\right) \sum_{i=1}^{n} Z_{i}^{(n)} \stackrel{p}{\rightarrow} 0 .
$$

This completes the proof.

Acknowledgments. The authors wish to the thank the anonymous referee, whose comment lead to improvements in the presentation of this paper. The research of M. Grabchak was supported, in part, by funds provided by the University of North Carolina at Charlotte.

\section{REFERENCES}

1. Абрамовии М., Стиган И. Справочник по специальным функциям с формулами, графиками и математическими таблицами. М.: Наука, 1979, 830 с.

2. Barczy M., Pap G. Portmanteau theorem for unbounded measures. - Statist. Probab. Lett., 2006, v. 76, № 17, p. 1831-1835.

3. Ben Arous G., Bogachev L. V., Molchanov S. A. Limit theorems for sums of random exponentials. - Probab. Theory Related Fields, 2005, v. 132, № 4, p. 579-612.

4. Bianchi M.L., Rachev S.T., Kim Y.S., Fabozzi F.J. Tempered infinitely divisible distributions and processes. - Теория вероятн. и ее примен., 20011, т. 55, в. 1, c. $59-86$.

5. Billingsley P. Probability and Measure. New York: Wiley, 1995, 593 p.

6. Bingham N. H., Goldie C. M., Teugels J. L. Regular Variation. Cambridge: Cambridge Univ. Press, 1987, $491 \mathrm{p}$.

7. Bogachev L. V. Extreme value theory for random exponentials. Probability and Mathematical Physics: A Volume in Honor of Stanislav Molchanov. Ed. by D. A. Dawson, V. Jaksic, and B. Vainberg. Providence: Amer. Math. Soc., 2007, p. 41-64. (CRM Proc. Lecture Notes, v. 42.)

8. Bovier A., Kurkova I., Löwe M. Fluctuations of the free energy in the REM and the p-spin SK model. - Ann. Probab., 2002, v. 30, № 2, p. 660-651.

9. Brockmann D., Hufnagel L. The scaling law of human travel - A message from George. - Complex Population Dynamics: Nonlinear Modeling in Ecology, Epidemiology and Genetics. Ed. by B. Blasius, J. Kurths, and L. Stone. Singapore: World Scientific Publ., 2007, p. 109-127.

10. Bullock J.M., Clarke R.T. Long distance seed dispersal by wind: measuring and modelling the tail of the curve. - Oecologia, 2000, v. 124, № 4, p. 506-521.

11. Chakrabarty A., Meerschaert M. M. Tempered stable laws as random walk limits. Statist. Probab. Lett., 2011, v. 81, № 8, p. 989-997.

12. Chakrabarty A., Samorodnitsky G. Understanding heavy tails in a bounded world or, is a truncated heavy tail heavy or not? - Stoch. Models, 2012, v. 12, № 1, p. 109-143.

13. Cont R., Tankov P. Financial Modelling with Jump Processes. Boca Raton: Chapman \& Hall, 2004, 535 p.

14. Cranston M., Molchanov S. A. Limit laws for sums of products of exponentials of i.i.d. random variables. - Israel J. Math., 2005, v. 148, № 1, p. 115-136.

15. Derrida B. Random-energy model: Limit of a family of disordered models. - Phys. Rev. Lett., 1980, v. 45, № 2, p. 79-82.

16. Феллер У. Введение в теорию вероятностей и ее приложения, т. 2. М.: Мир, 1984, $752 \mathrm{c}$.

17. Feng Y., Molchanov S., Whitmeyer J. Random walks with heavy tails and limit theorems for branching processes with migration and immigration. - Stoch. Dyn., 2012, v. 12, № 1, paper № 1150007, 23 p. 
18. Grabchak M. Limit theorems for sequences of tempered stable and related distributions. arXiv:1201.6006.

19. Grabchak $M$. On a new class of tempered stable distributions: moments and regular variation. - J. Appl. Probab., 2012, v. 49, № 4, p. 1015-1035.

20. Grabchak M. Inversions of Lévy measures and the relation between long and short time behavior of Lévy processes. - J. Theoret. Probab., 2013, DOI 10.1007/s10959012-0476-6.

21. Грабиак M., Малчанов C.A. Предельные теоремы и фазовые переходы для двух моделей суммирования независимы одинаково распределенных случайных величин, зависящих от параметров. - Докл. РАН, 2013, т. 451, № 4, с. 374-377.

22. Grabchak M., Samorodnitsky G. Do financial returns have finite or infinite variance? A paradox and an explanation. - Quant. Finance, 2010, v. 10, № 8, p. 883-893.

23. Gut A. Stopped Random Walks: Limit Theorems and Applications. New York: Springer, 2009, $263 \mathrm{p}$.

24. Ibragimov I. A., Linnik Yu. V. Independent and Stationary Sequences of Random Variables. Gröningen: Wolters-Noordhoff, 1971, 443 p.

25. Ибрагимов И.А., Пресман Э.Л. О скорости сближения распределений сумм независимых случайных величин с сопровождающими законами. - Теория вероятн. и ее примен., 1973, т. 18, в. 4, с. 753-766.

26. Jørgensen B., Martinez J. R., Vinogradov V. Domains of attraction to Tweedie distributions. - Lith. Math. J., 2009, v. 49, № 4, p. 399-425.

27. Kondratiev Y., Kutoviy O., Pirogov S. Correlation functions and invariant measures in continuous contact model. - Infin. Dimens. Anal., Quantum Probab. Relat. Top., 2008, v. 11, № 2, p. 231-258.

28. Le Cam L. On the distribution of sums of independent random variables. Bernoulli 1713 Bayes 1763 Laplace 1813: Anniversary Volume. Ed. by J. Neyman and L. Le Cam. Berlin-New York: 1965, p. 179-202.

29. Meerschaert M. M., Scheffler H. Limit Distributions for Sums of Independent Random Vectors: Heavy Tails in Theory and Practice. Chichester: Wiley, 2001, 484 p.

30. Resnick S.I. Heavy-Tail Phenomena: Probabilistic and Statistical Modeling. New York: Springer, 2007, 404 p.

31. Rosiński J. Tempering stable processes. - Stochastic Process. Appl., 2007, v. 117, № 6, p. 677-707.

32. Sato K. Lévy Processes and Infinitely Divisible Distributions. Cambridge: Cambridge Univ. Press, 1999, 486 p.

33. Зайщев А.Ю., Арак Т.В. О скорости сходимости во второй равномерной предельной теореме Колмогорова. - Теория вероятн. и ее примен., 1983, т. 28, в. 2 , с. $333-353$.

34. Зайцев А.Ю. О равномерной аппроксимации функций распределения сумм независимых случайных величин. - Теория вероятн. и ее примен., 1987, т. 32 , B. 1 , c. $45-52$.

35. Зайщев А.Ю. Многомерный вариант второй равномерной предельной теоремы Колмогорова. - Теория вероятн. и ее примен., 1989, т. 34 , в. 1, с. 128-151.

36. Зайчев А. Ю. Аппроксимация сверток вероятностных распределения безгранично делимыми законами при ослабленных моментных ограничениях. - Зап. научн. сем. ЛОМИ, 1992, т. 194, с. 79-90.

Поступила в редакцию

5.I.2013

Исправленный вариант

10.XII.2013 